\title{
CRITICAL HEAT FLUX EXPERIMENTS WITH A LOCAL HOT PATCH IN AN INTERNALLY HEATED ANNULUS \\ (LWBR Development Program)
}

E. P. MORTIMORE

S. G. BEUS

FEBRUARY 1979

CONTRACT EY-76-C-11-0014

BETTIS ATOMIC POWER LABORATORY WEST MIFFLIN, PENNSYLVANIA

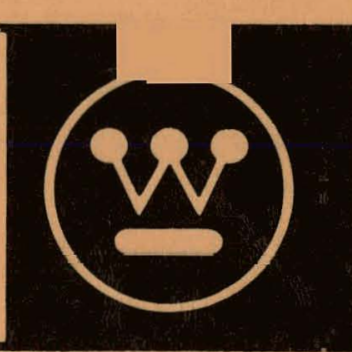




\section{DISCLAIMER}

This report was prepared as an account of work sponsored by an agency of the United States Government. Neither the United States Government nor any agency Thereof, nor any of their employees, makes any warranty, express or implied, or assumes any legal liability or responsibility for the accuracy, completeness, or usefulness of any information, apparatus, product, or process disclosed, or represents that its use would not infringe privately owned rights. Reference herein to any specific commercial product, process, or service by trade name, trademark, manufacturer, or otherwise does not necessarily constitute or imply its endorsement, recommendation, or favoring by the United States Government or any agency thereof. The views and opinions of authors expressed herein do not necessarily state or reflect those of the United States Government or any agency thereof. 


\section{DISCLAIMER}

Portions of this document may be illegible in electronic image products. Images are produced from the best available original document. 
CRITICAL HEAT FLUX EXPERIMENTS WITH

A LOCAL HOT PATCH IN AN INTERNALLY HEATED ANNULUS

(LWBR Development Program)

E. P. Mortimore

S. G. Beus

Contract No.: EY-76-C-11-0014

FEBRUARY 1979

Printed in the United States of America

Available from the

National Technical Information Service

U. S. Department of Commerce

5285 Port Royal Road

Springfield, Virginia 22151

NOTE

This report was prepared as an account of work sponsored by the United States Govemment. Neither the United States nor the United States Department of Energy, nor any of their employees, nor any of their contractors, subcontractors, or theiz employees, makes any warranty, express or implied, or assumes any legal liability or responsibility for the accuracy, completenese or usefulness of any information, apparatus, product or process disclosed, or represents that its use would not infringe privately owned rights.

This document is an interim memorandum prepared primarily for internal reference and does not represent a final expression of the opinion of Westinghouse. When this memorandum is distributed externally, it is with the express understanding that Westinghouse makes no representation as to completeness, accuracy, or usability of information contained herein. 
This report was prepared as an account of work sponsored by the United States Government. Neither the United States, nor the United States Department of Energy, nor any of their employees, nor any of theit contractors, subcontractors, or their employees, makes any warranty, express or implied, or assumes any legal liability or responsibility for the accuracy, completeness or usefulness of any information, appuratus, product or process disclosed, or represents that its use would not infringe privately owned rights. 
The Shippingport Atomic Power Station located in Shippingport, Pennsylvania was the first large-scale, central-station nuclear power plant in the United States and the first plant of such size in the world operated solely to produce electric power. This program was started in 1953 to confirm the practical application of mucicar power for large-scale electric power generation. It has provided much of the technology being used for design and operation of the comercial, centralstation nuclear power plants now in use.

Subsequent to development and successful operation of the Pressurized Water Reactor in the DOE-owned reactor plant at the Shippingport Atomic Power Station, the Atomic Energy Commission in 1965 undertook a research and development program to design and build a Light Water Breeder Reactor core for operation in the Shippingport Station.

The objective of the Light Water Breeder Reactor (IWBR) program has been to develop a technology that would significantly improve the utilization of the nation's nuclear fuel resources employing the well-established water reactor technology. To achieve this objective, work has been directed toward analysis, design, component tests, and fabrication of a water-cooled, thorium oxide fuel cycle breeder reactor for installation and operation at the Shippingport Station. The LWBR core started operation in the Shippingport Station in the Fall of 1977 and is expected to be operated for about 3 to 4 years. At the end of this period, the core will be removed and the spent fuel shipped to the Naval Reactors Expended Core Facility for a detailed examination to verify core performance including an evaluation of breeding characteristics.

In 1976, with fabrication of the Shippingport IWBR core nearing completion, the Energy Research and Development Administration established the Advanced Water Breeder Applications (AWBA) program to develop and disseminate technical information which would assist $U$. S. Industry in evaluating the IWBR concept for commercial-scale applications. The program will explore some of the problems that would be faced by industry in adapting technology confirmed in the IWBR program. Information to be developed includes concepts for commercial-scale prebreeder cores which would produce uranium-233 for light water breeder cores while producing electric power, improvements for breeder cores based on the technology developed to fabricate and operate the Shippingport LWBR core, and other information and technology to aid in evaluating commercial-scale application of the LWBR concept.

All three development programs (Pressurized Water Reactor, IIght Water Breeder Reactor, ind Advanced Water Breeder Applications) have been administered by the Division of Naval Reactors with the goal of developing practical improvements in the utilization of nuclear fuel resources for generation of electrical energy using water-cooled nuclear reactors.

Technical information developed under the Shippingport, LWBR, and AWBA programs has been and will continue to be published in technical memoranda, one of which is this present report. 
SECTION

FOREWORD

ABSTRACT

I. INTRODUCTION

II. TEST EQUIPMENT 2 . 2

III. TEST PROCEDURE $\quad 4$

IV. DISCUSSION OF RESULTS

A. Hot Patch Data Comparison with Flux Ratio of 1.50

B. Hot Patch Data Comparison with a Flux Ratio of. 2.25

V. CONCLUSIONS

References

TABLE 1 - Comparison of Data With and Without. A Hot Patch - 1.5 Heat Flux Ratio

TABLE 2 - Comparison of Data With and Without A Hot Patch - 2.25 Heat Flux Ratio

FIGURE 1 - Schematic Diagram of Hot Patch Test Loop

FIGURE 2 - Test Section and Instrumentation

FIGURE 3 - Partial Axial Veiw of Hot Patch Test Section, Ceramic Housing and Backup Housing

FIGURE 4 - Full Axial View of Hot Patch Test Section,

FIGURE 5 - Detailed Schematic of Inlet Region of Hot Patch Test Section

FIGURE 6 - Heated Tube Exit End Connection Detail (Not to Scale)

FIGURE 7 - CHF Ratio Data Comparison for Hot Patch Test with 1.5 Heat Flux Ratio

FIGURE 8 - CHF Ratio Data Comparison for 1.5 Hot Patch Test Heat Flux Ratio

FIGURE 9 - CHF Ratio Data Comparison for 1.5 Hot Patch Test

FIGURE 10- Summary of A11 CHF Data at 2000 psia

FIGURE 11- Summary of A11 CHF Data at 1600 psia

FIGURE 12- Summary of All CHF Data at 1200 psia

FIGURE 13- CHF Ratio Data Comparison for 2.25 Hot Patch Test

FIGURE 14- CHF Ratio Data Comparison for 2.25 Hot Patch Test

FIGURE 15- CHF Ratio Data Comparison for 2.25 Hot Patch Test 
Critical heat flux experiments were conducted for upflow of water in a vertical 84 inch annular flow channel, 0.303 inch heated I.D. and 0.500 inch unheated $0 . D$. Test data were obtained at pressures from 1200 to 2000 psia, mass velocities from $0.25 \times 10^{6}$ to $2.8 \times 10^{6} \mathrm{lb} / \mathrm{hr}-\mathrm{ft}^{2}$ and inlet temperatures ranging from 200 to $600^{\circ} \mathrm{F}$. Three different test sections were employed with (1) axially uniform heat flux over the 84 inch length to serve as a no-hot-patch data base, (2) axially uniform heat flux over 82 inches with a 1.5 heat flux ratio hot patch over the last two inches, and (3) axially uniform heat flux over 82 inches with a 2.25 heat flux ratio hot patch over the last two inches. Comparisons of hot patch to no-hot-patch critical heat flux values were made indicating that for most cases a uniform two-inch exit hot patch does not cause a reduction in $\mathrm{CHF}$ at either hot patch ratio. However, there was a tendency for a CHF decrement to occur for the low inlet enthalpy runs at high mass velocity.

CRITICAL HEAT FLUX EXPERIMENTS WITH

A LOCAL HOT PATCH IN AN INTERNALLY HEATED ANNULUS (LWBR Development Program)

\section{INTRODUCTION}

Local heat flux peaking can occur inadvertently in nuclear reactor cores having fuel rods due to the combined effects of fuel pellet eccentricity, non-uniform pellet porosity and potential fuel stack gaps created by densification during core power operation. Any one or combination of these conditions can produce a local region in. a fuel rod which operates at a heat flux higher than that intended, thus increasing the chance for the occurrence of a critical heat flux (CHF) condition in that region.

The local conditions hypothesis of critical heat flux phenomena suggests that the increased heat flux over a region in a fuel rod could produce a CHF condition in the rod at a lower average rod heat flux compared to a fuel rod without local flux peaking. However, previous experimental evidence suggests that if the size of the region and/or the magnitude of the heat flux peak is small enough then the local flux 
peak region will have no effect on CHF (Reference 1).

Reference 1 reports results from an experiment conducted to determine the effect of a hot patch at the end of the heated length on CHF power levels. The test section was the same as that employed in the subject experiment except that the inner diameter of the electrically heated tube was reamed out for the last $1-1 / 4$ inches to produce a circumferentially uniform hot patch with a heat flux 1.337 times the rod average surface heat flux. Direct comparison of the CHF power levels of this test section with those of a similar test section without the hot patch indicated that the hot patch did not affect the CHF power capability of the rod.

A comprehensive test program for hot patch effectiveness is discussed in Reference 2 which presents experimental data obtained over a range of hot patch lengths from 0.591 inches to 7.874 inches and with heat flux peaking factors of 1.5 and 2.0. The test sections employed in these tests were electrically heated $8 \mathrm{~mm}$ I.D. tubes with water flowing on the inside. The subject test program was undertaken to provide supplementary data concerning the effect of hot patches. In particular, available hot patch data in the literature were lacking at low qualities (less than $8 \%$ ) where previous data obtained at Bettis indicated potential effects of hot patch on thermal pertormance.

\section{TEST EQUIPMENT}

The test equipment consisted of a high pressure water circulation loop, a D-C generator, the test section, local instrumentation and the data acquisition system. A Crocker-Wheeler direct current generator was used for test section electrical power with maximum ratings of 1.00 volts and 7500 amps. Additional test section current can be obtained by arranging up to four generators in parallel. The tests were performed in Bettis High Pressure Loop No. 29 shown schematically in Figure 1. The loop can circulate $50 \mathrm{gpm}$ of water through 2 inch 316 stainless steel piping at pressures up to $2500 \mathrm{psia}$ and $650^{\circ} \mathrm{F}$ using a single canned motor pump.

The test section was a $0.303 \pm 0.001$ inch $0 . D$. stainless steel tube with a 0.035 inch thick wall, surrounded by a 0.500 inch I.D. ceramic tube contained within a 1 inch O.D. pipe (see Figure 2). An annular flow path exists between the inside 0.303 inch $0 . D$. tube and the 0.500 inch 
I.D. ceramic tube. The ceramic tube and outer pipe are in contact. The inside of the heated tube contains air at atmospheric pressure. The heated tube was centered within the ceramic tubing by seven spacers located at 12 inch intervals with the uppermost spacer located 9 inches before the end of the heated length. Each spacer consisted of three short tubing segments welded to the test section at $120^{\circ}$ locations as can be seen in Figure 3. Copper terminals are attached to both ends of the tube and a heat flux is produced by DC resistance heating in the tube wall. Heat flux from the tube wall was uniform axially except for the increased heat flux in the 2 inch hot patch which was produced by reaming out the inside of the stainless steel tube to reduce the wall thickness to 0.026 inch at the end, thus increasing the electrical resistance locally.

Figure 2 shows an axial and cross-sectional view of the test section identifying the location of the outlet wall thermocouples and the dimensions of the annular flow channel. Figures 3 and 4 give partial and full length axial views of the disassembled test section. Figures 5 and 6 show detailed drawings of the inlet and exit regions of the test section showing the inlet pressure tap and the design of the heated tube and terminal connections. The test section was well insulated and heat losses were negligible throughout the ful1 range of testing.

Stainless steel sheathed chromel-alumel thermocouples were used for water temperature indication. Four water the rmocouples were positioned in the flow, two upstream and two downstream of the heated length. Two asbestos-insulated chromel-alumel wall thermocouples were spot welded inside the heated tube near the exit end of the test section as shown in Figure 6. Readouts from these thermocuples were displayed continuously on oscillograph charts and were used to indicate $\mathrm{CHF}$. CHF was recognized by a $2 \mathrm{~mm}$ or greater rise of either $\mathrm{CHF}$ thermocouple oscillograph reading from its base line value corresponding to a temperature rise of $20^{\circ} \mathrm{F}$.

Power measurements were recorded continuously as voltage drop across the test section terminals and current as measured by millivolt

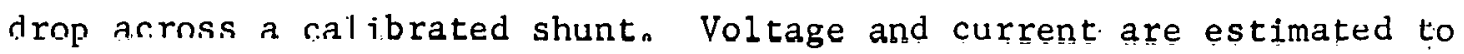
be accurate to within $\pm 1.0 \%$ and $\pm 0.8 \%$ respectively.

High purity water was used in all tests with a $\mathrm{pH}$ near 7.0. The resistivity was maintained above 0.5 megohm $-\mathrm{cm}$ at room temperature and the oxygen concentration was ma1ntained below $0.1 \mathrm{ppm}$. 
The test section flow rate was measured with two orifices of the same size connected in series. Orifices of diameters $0.100,0.300$ and 0.435 inch were used in three parallel flow legs. The size used for a given test run depended on the flow rate. All orifice calibration constants were evaluated from tests with a weigh tank and flowrates calculated from the two orifices agreed within $2 \%$, Water temperature at the orifices was measured by two thermocouples accurate to about $\pm 2{ }^{\circ} \mathrm{F}$.

The mass velocities were calculated using the measured flowrates and the nominal flow area of the test section. There is a significant uncertainty $(+6 \%,-34 \%)$ in local mass velocity due to the inner diameter tolerance of the ceramic tubing. This uncertainty is not present in the local enthalpy since this quantity is calculated from the test section power and flowrate determined from orifice readings.

The steady-state data acquisition system consisted of oscillograph recorders for CHF thermocouple monitoring and strip chart recorders for generator current and hub-to-hub test section voltage drop. The oscillographs were electrically coupled to the test section power supply such that test section power was automatically reduced by $44 \%$ when a $\mathrm{CHF}$ temperature excursion was indicated.

An Integrating Digital Voltmeter (IDVM) was used to measure all thermocouple and Differential Pressure (DP) cell readings. These readings were saved on magnetic tape recordings.

Water tlow rates were determined as the numerical average of values calculated from the two orifices connected in series. The test section inlet mass velocity was determined by dividing the flowrate by the test section inlet flow area. The measurement error on the inlet temperature is estimated at $\pm 2^{\circ} \mathrm{F}$. For the system pressure, the estimated error is \pm 3 psi while the heat flux and inlet mass velocity are each estimated to be accurate within $\pm 2 \%$. These errors combined with the uncertainty inherent in identifying CHF lead to an estimated $\pm 10 \%$ allowed error band on a computed hot patch to no-hot-patch flux ratio.

\section{TEST PROCEDURE}

The test procedures for the no-hot-patch, 1.5 flux ratio hot patch and the 2.25 flux ratio hot patch CHF tests were identical and consisted of: 
1. Establishing loop conditions of pressure, flowrate, chemistry $(\mathrm{pH}=7.0)$ and test section inlet temperature.

2. Applying and increasing the test section power to an estimated value below $\mathrm{CHF}$ and increasing power in smal1 increments until a CHF indication was obtained on either of the exit wall thermocouple oscillograph charts.

3. Recording al1 data.

Following a CHF run, the power was reset to approximately $98 \%$ of the $\mathrm{CHF}$ power level and a complete line of data recorded. These $98 \%$ runs served as a backup indication of nominal test section conditions for the CHF runs where a rapid $\mathrm{CHF}$ prevented the recording of a full line of data on the Integrating Digital Voltmeter (IDVM).

IV. DISCUSSION OF RESULTS

The data obtained at $\mathrm{CHF}$ and at $98 \%$ of $\mathrm{CHF}$ from the 1.5 Hot Patch. Test are contained in Table 1. Comparable data for the 2.25 Hot Patch Test are contained in Table 2. The CHF data with no hot patch are shown for comparison in both tables. Each table lists the run number, run type, nominal system pressure, inlet mass velocity, inlet temperature, inlet enthalpy, calculated average exit enthalpy, average test section heat flux and the ratio of average heat fluxes with and without the hot patch. The quantities given in these tables are the actual test conditions.

The exit enthalpy was calculated from the equation:

$$
H_{\text {exit }}(B t u / 1 b)=H_{\text {in }}+\frac{Q^{\prime \prime} A_{h}}{G A_{f}}
$$

where

$$
\begin{aligned}
\mathrm{H}_{\mathrm{in}} & =\text { inlet enthalpy }(\mathrm{Btu} / \mathrm{lb}) \\
\mathrm{Q}^{\prime \prime} & =\text { average heat flux }\left(\mathrm{Btu} / \mathrm{hr}-\mathrm{ft}^{2}\right) \\
G & =\text { test section mass velocity }\left(1 \mathrm{~b} / \mathrm{hr}-\mathrm{ft}^{2}\right) \\
A_{\mathrm{h}} & =\text { test section heat transfer area }=0.5553 \mathrm{ft}^{2} \\
A_{f} & =\text { test section annular flow area }=0.0008625 \mathrm{ft}^{2}
\end{aligned}
$$


Any effect of the hot patch on critical heat flux performance can be deduced by comparing the heat flux of pairs of runs in l'ables 1 and 2. If the inlet fluid conditions for any pair of runs are very close then the heat fluxes can be compared directly. If the inlet fluid conditions for any pair of runs differ significantly, then allowance must be made for the different fluid conditions.

The heat flux ratios printed in these tables were used to develop plots discussed below. In some cases the $98 \% \mathrm{CHF}$ points were used on the plots and these are indicated in the tables.

No comparison should be made between runs 34 and 158 at CHF or rune 35 and 159 at $98 \% \mathrm{CHF}$ because runs 34 and 35 had $\mathrm{T}_{\text {in }} \approx 200^{\circ} \mathrm{F}$ while runs 150 dul 159 liad $\mathrm{T}_{\text {In }} \approx 427^{\circ} \mathrm{F}$ due to generator limitationo which occurred during runs 158 and 159.

\section{A. Hot Patch Data Comparison with Flux Ratio of 1.50}

Figure 7 summarizes the results of the 1.5 flux ratio hot patsh testing as compared to the no-hot-patch data base (referrcd to as "new") at 2000,1600 and 1200 psia. The results are presented in tabular form in Table 1. The deviation of the hot patch CHF data from the no-hotpatch data base may be characterized by the CHF Ratio, defined as the ratio of the average heat flux at $\mathrm{CHF}$ with hot patch to the average heat flux at 'HF' without hot patch for the same pressure, mass velocity and inlet temperature. An examination of the data in Figure 7 shows that there is a decided mass velocity effect and that the CHF ratio is between 0.90 and 1.10 except for six of the 37 data points:

$$
\begin{aligned}
& \text { a. } 2000 \mathrm{psia}, 200^{\circ} \mathrm{F}, 0.25 \times 10^{6} \mathrm{lb} / \mathrm{hr}-\mathrm{ft}^{2} \text {, Ratio }=1.12 \\
& \text { b. } 1600 \mathrm{psia}, 400^{\circ} \mathrm{F}, 0.25 \times 10^{6} \mathrm{lb} / \mathrm{hr}-\mathrm{ft}^{2} \text {, Ratio }=1.11 \\
& \text { c. } 1600 \mathrm{psia}, 500^{\circ} \mathrm{F}, 0.25 \times 10^{6} \mathrm{lb} / \mathrm{hr}-\mathrm{ft}^{2} \text {, Ratio }=1.11 \\
& \text { d. } 1200 \mathrm{psia}, 200^{\circ} \mathrm{F}, 0.25 \times 10^{6} \mathrm{lb} / \mathrm{hr}-\mathrm{ft}^{2} \text {, Ratio }=1.26 \\
& \text { e. } 1200 \mathrm{psia}, 400^{\circ} \mathrm{F}, 0.25 \times 10^{6} \mathrm{lb} / \mathrm{hr}-\mathrm{ft}^{2} \text {, Ratio }=1.17 \\
& \text { f. } 1200 \mathrm{psia}, 500^{\circ} \mathrm{F}, 0.25 \times 10^{6} \mathrm{lb} / \mathrm{hr}-\mathrm{ft}^{2} \text {, Ratio }=1.16 \\
& \text {, R }
\end{aligned}
$$


It is noted that all six of these points are at $0.25 \times 10^{6}$ $1 \mathrm{~b} / \mathrm{hr}-\mathrm{ft}^{2}$. inlet mass velocity and the ratio is always greater than 1.00 . Since it is not expected that the hot patch would cause an improvement in $\mathrm{CHF}$ at $0.25 \times 10^{6} \mathrm{bb} / \mathrm{hr}-\mathrm{ft}^{2}$ mass velocity, it is concluded that these points are probably in error. It may also be concluded from these comparisons that the 1.5 flux ratio hot patch does not result in earlier CHF.

Because of the unrealistic trend of the test results discussed above at low mass velocities, an alternate set of base case data obtained with a nominally identical test section in a previous test (as described in the Appendix)was utilized. Figure 8 summarizes the results of the 1.5 flux ratio hot patch testing at 2000 and 1200 psia using the al ternate data base referred to in the Appendix as the "first assembly". Figure 8 shows that all the CHF ratios at 2000 psia are within the $\pm 10 \%$ experimental scatter band while three points at 1200 psia are outside the scatter band. Again, however, all of the CHF ratios outside of the scatter band are greater than 1.00 suggesting no hot patch CHF decrements.

Figure 9 compares the results of the 1.5 flux ratio hot patch testing at 2000,1600 and 1200 psia, with a second alternate data set from a previous test. These data were obtained in a nominally identical test section referred to in the Appendix as the "second assembly". Figure 9 shows that all the CHF ratios at all pressures are within the $\pm 10 \%$ experimental scatter band.

Figures 10, 11 and 12 compare the results of the 1.5 heat flux hot patch testing to the no-hot-patch testing on the conventional fluxenthalpy plot at 2000,1600 and 1200 psia, respectively. The 1.5 hot patch data are represented by open squares while the no-hot-patch data bases are represented by circles. An examination of these three figures shows that the 1.5 hot patch data generally lie very close to the no-hot-patch data and are consistently above and to the right of the no-hot-patch data at the lower mass velocities. A comparison of the old and new no-hot-patch data bases indicates a probable experimental bias between the two sets of data. l'he only known difference between the old no-hot-patch test 
section and the new no-hot-patch test section was the type of spacers used to center the stainless steel test tube in the two tests. The old test used "spring collar" spacers described in the Appendix while the new test section used small diameter open tubes which were located at the same axial locations as the spring collars thus yielding a nominally centered test tube in both cases. In both cases there was some unknown experimental tolerance in centering the tube, thus leading to the possibility that the one test section tube was more eccentric than the other. However, it is not known if differences in test section eccentricity could introduce the observed differences in the two data bases.

Two conclusions can be drawn from a comparison of the $1.5 \mathrm{CHF}$ ratio data based on the new no-hot-patch data base vs. the same CHF ratio based on the old (Appendix) no-hot-patch data base:

1. The CHF ratio is sensitive to the data base used, especially at $0.25 \times 10^{6} \mathrm{lb} / \mathrm{hr}-\mathrm{ft}^{2}$.

2. The CHF ratio is never less than 0.9 irrespective of the no-hot-patch data base used, strongly suggesting that there is no-hot-patch $\mathrm{CHF}$ decrement over the range of variables tested.

B. Hot Patch Data Comparison with a Flux Ratio of 2.25

Flgure 13 summarizes the results of the current 2.25 flux ratio hot patch testing at 2000, 1600 and 1200 psia, as compared with the new no-hot-patch data base. These data are tabulated in Table 2. An examination of these data shows that the hot patch to no-hot-patch CHF ratio is between 0.90 and 1.10 except for six of the 34 data points:

a. $2000 \mathrm{psia}, 600^{\circ} \mathrm{F}, 0.25 \times 10^{6} \mathrm{lb} / \mathrm{hr} \mathrm{ft} \mathrm{t}^{2}$, CHF Ratio $=1.15$

b. 2000 psia, $200^{\circ} \mathrm{F}, 1.00 \times 10^{6} \mathrm{lb} / \mathrm{hr}-\mathrm{ft}^{2}$, CHF Ratio $=0.86$

e. LUUU psia, $500^{\circ} \mathrm{F}, 2.00 \times 10^{6} \mathrm{lb} / \mathrm{hr}-\mathrm{ft}{ }^{2}, \mathrm{CHF}$ Ratio $=0.88$

d. 1200 psia, $200^{\circ} \mathrm{F}, 0.25 \times 10^{6} \mathrm{lb} / \mathrm{hr}-\mathrm{ft}^{2}$, $\mathrm{CHF}$ Ratio $=1.16$

e. $1200 \mathrm{psia}, 400^{\circ} \mathrm{F}, 2.00 \times 10^{6} \mathrm{lb} / \mathrm{hr}-\mathrm{ft}^{2}$, CHF Ratio $=0.80$

f. $1200 \mathrm{p} \varepsilon \mathrm{ia}, 500^{\circ} \mathrm{F}, 1.00 \times 10^{6} \mathrm{lb} / \mathrm{hrmt}^{2}$, CIIF Ratio $=0.00$

It is noted that the two points at $0.25 \times 10^{6} \mathrm{lb} / \mathrm{hr}-\mathrm{ft}^{2}$ are both greater than 1.10 while there are four data points for which a CHF decrement exists. 
Figure 14 summarizes the results of current 2.25 hot patch testing at 2000 and $1200 \mathrm{psia}$, using the alternate data base. The no-hot-patch heat flux values used to compute the CHF ratios for these figures were taken from a previous test and were obtained on a nominally identical test section referred to in the Appendix as the "first assembly".

Figure 14 shows that all but one of the CHF ratios at 2000 psia are within the $\pm 10 \%$ scatter band while three points at 1200 psia are outside the scatter band. However, only one point is on the low side of the scatter band at conditions of $1200 \mathrm{psia}, 400^{\circ} \mathrm{F}$ and $2.0 \times 10^{6} 1 \mathrm{~b} / \mathrm{hr}-\mathrm{ft}^{2}$ where the $\mathrm{CHF}$ ratio is 0.87 .

Figure 15 summarizes the results of current 2.25 hot patch testing at 2000,1600 and $1200 \mathrm{psia}$, with a second alternate data base from a previous test. The no-hot-patch heat flux values used to compute the CHF ratios for these figures were obtained on a nominally identical test section referred to in the Appendix as the "second assembly". This comparison shows that all of the CHF ratios at all pressures are within the $\pm 10 \%$ experimental scatter band.

Figures 10, 11 and 12 compare the results of the 2.25 hot patch testing to the no-hot-patch testing results on the conventional fluxenthalpy plot at 2000, 1600 and 1200 psia, respectively. The 2.25 hot patch data are represented by open triangles while the no-hot-patch data base is represented by circles. An examination of these figures shows that the 2.25 hot patch data generally lie close to the no-hot-patch data and all but one ( $1200 \mathrm{psia}, 400^{\circ} \mathrm{F}, 2.00 \times 10^{6} \mathrm{lb} / \mathrm{hr}-\mathrm{ft} \mathrm{t}^{2}$ ) of the 2.25 hot patch data points are judged to lie within the experimental scatter band of the comparable old no-hot-patch data point. At the higher mass velocities the re appears to be a trend of the 2.25 hot patch data to lie below and to the left of the no-hot-patch data base and this may suggest a emall CHF decrement at these conditions.

\section{CONCLUSIONS}

The 1.5 hot patch to no-hot-patch CHF ratio is always greater than 0.90, independently of the no-hot-patch data base (old and new) used to compute the CHF ratio. This result indicates that there is no hot patch 
CHF decrement due to a 2 inch long 1.5 flux ratio hot patch over the range of variables and geometry tested.

The 2.25 hot patch to no-hot-patch CHF ratio, based on the new data base, is greater than 0.90 except for data at four conditions:

a. 2000 psia, $200^{\circ} \mathrm{F}, 1,000,000 \mathrm{lb} / \mathrm{hr}-\mathrm{ft}^{2}, \mathrm{CHF}$ Ratio $=0.86$

b. $2000 \mathrm{psia}, 500^{\circ} \mathrm{F}, 2,000,000 \mathrm{lb} / \mathrm{hr}-\mathrm{ft}{ }^{2}$, CHF Ratio $=0.88$

c. $1200 \mathrm{psia}, 500^{\circ} \mathrm{F}, 1,000,000 \mathrm{Ib} / \mathrm{hr}-\mathrm{ft}^{2}$, CHF Ratio $=0.88$

d. $1200 \mathrm{psia}, 400^{\circ} \mathrm{F}, 2,000,000 \mathrm{lb} / \mathrm{hr}-\mathrm{ft}{ }^{2}$, $\mathrm{CHF}$ Ratio $=0.80$

The 2.25 hot patch to no-hot-patch CHF ratio, based on the old (Appendix) data base, is greater than 0.90 except for data at one condition:

$$
1200 \text { psia, } 400^{\circ} \mathrm{F}, 2,000,000 \times 10^{8} \mathrm{lb} / \mathrm{hr}-\mathrm{ft}^{2} \text {, CHF Ratio }=0.87
$$

These results indicate that there is no CHF decrement for most of the hot patch data with a heat flux ratio of 2.25 over the range of variables tested. However, there was a tendency for a CHF decrement to occur for the low inlet enthalpy runs at high mass velocity.

Based on a comparison of the new no-hot-patch data with the old no-hot-patch data (Appendix), the new no-hot patch data at $250,000 \mathrm{lb} / \mathrm{hr}-\mathrm{ft}^{2}$ mass velocity are probably in error. If these data are used, there is a consistent trend in both the hot patch data sets showing an improvement in $\mathrm{CHF}$ due to the hot patch at $250,000 \mathrm{lb} / \mathrm{hr}-\mathrm{ft}^{2}$. This trend is not evident if the old no-hot-patch data are used.

\section{Refertuces}

1. WAPD-TM-1154, "Effect of Axial Locations of Spacers and Hot Spots on the CHF Power of Rods," K. J. Coeling, September 1978

2. V. N. Smolin, et al, "Departure from Nucleate Boiling in Channels with Heat Flux Peaking," Thermal Engineering, Vo1. 17, No. 5, May 1970

3. WAPD-TM-1013, "Critical Heat F1ux and Pressure Drop Tests in Bundles of Twenty Rods," B. W. Le'lourneau, et al, January 1975 
TABLE 1

Comparison of Data With and Without A Hot Patch -1.5 Heat Flux Ratio

\begin{tabular}{|c|c|c|c|c|c|c|c|c|c|c|}
\hline $\begin{array}{l}\text { RUN } \\
\text { NO. }\end{array}$ & & TYPE & & $\begin{array}{l}\text { PRES- } \\
\text { (PSIA) }\end{array}$ & $\begin{array}{l}\text { MASS } \\
\text { VELOCITY } \\
X 10-5 \\
\text { (LB/HR- } \\
\text { FTSOI }\end{array}$ & $\begin{array}{c}\text { INLET } \\
\text { TEMPER- } \\
\text { ATURE } \\
\text { (DEG.F.I }\end{array}$ & $\begin{array}{l}\text { MEASURED } \\
\text { INLET } \\
\text { ENTHALPY } \\
\text { (BTU/L B) }\end{array}$ & $\begin{array}{l}\text { CALCU- } \\
\text { LATEO } \\
\text { EXIT } \\
\text { ENTHALPY } \\
\text { (BTU/LB) }\end{array}$ & $\begin{array}{l}\text { AVERAGE } \\
\text { HEAT FLUX } \\
\text { X 1O-6 } \\
\text { (BTU/HR- } \\
\text { FTSQ I }\end{array}$ & $\begin{array}{l}\text { RATIO } \\
\text { OF } \\
\text { CRITICAL } \\
\text { HEAT } \\
\text { FLU XES }\end{array}$ \\
\hline $\begin{array}{l}38 \\
82\end{array}$ & $\begin{array}{l}\text { CHF } \\
\text { CHF }\end{array}$ & $\begin{array}{c}H / O \\
H\end{array}$ & $\begin{array}{l}H P \\
H P\end{array}$ & $\begin{array}{l}2000 \\
2000\end{array}$ & $\begin{array}{l}0.259 \\
0.253\end{array}$ & $\begin{array}{l}201.5 \\
199.8\end{array}$ & $\begin{array}{l}174.2 \\
172.4\end{array}$ & $\begin{array}{l}779.4 \\
856.4\end{array}$ & $\begin{array}{l}0.244 \\
0.269\end{array}$ & 1.10 \\
\hline $\begin{array}{l}36 \\
84\end{array}$ & $\begin{array}{l}\text { CHF } \\
\text { NO }\end{array}$ & $\begin{array}{l}\text { W/O } \\
\text { OATA }\end{array}$ & $H P$ & 2000 & 0.499 & 201.4 & 174.1 & 706.8 & 0.413 & \\
\hline $\begin{array}{r}34 \\
158\end{array}$ & $\begin{array}{l}\text { CHF } \\
\text { CHF }\end{array}$ & $H_{H}$ & $\begin{array}{l}\text { HP } \\
\text { HP }\end{array}$ & $\begin{array}{l}2000 \\
2000\end{array}$ & $\begin{array}{l}0.991 \\
1.001\end{array}$ & $\begin{array}{l}200.7 \\
427.8\end{array}$ & $\begin{array}{l}173.4 \\
407.3\end{array}$ & $\begin{array}{l}635.3 \\
707.7\end{array}$ & $\begin{array}{l}0.711 \\
0.467\end{array}$ & ** \\
\hline $\begin{array}{l}27 \\
85\end{array}$ & $\begin{array}{l}\text { CHF } \\
\text { CHF }\end{array}$ & $H_{H}^{H}$ & $\begin{array}{l}\text { HP } \\
\text { HP }\end{array}$ & $\begin{array}{l}2000 \\
2000\end{array}$ & $\begin{array}{l}0.245 \\
0.253\end{array}$ & $\begin{array}{l}401.8 \\
400.5\end{array}$ & $\begin{array}{l}379.2 \\
377.8\end{array}$ & $\begin{array}{l}858.3 \\
889.3\end{array}$ & $\begin{array}{l}0.182 \\
0.201\end{array}$ & 1.11 \\
\hline $\begin{array}{l}29 \\
99\end{array}$ & $\begin{array}{l}\text { CHF } \\
\text { CHF }\end{array}$ & W/O & $\begin{array}{l}\text { HP } \\
\text { HP }\end{array}$ & $\begin{array}{l}2000 \\
2000\end{array}$ & $\begin{array}{l}0.500 \\
0.497\end{array}$ & $\begin{array}{l}401.7 \\
400.0\end{array}$ & $\begin{array}{l}379.1 \\
377.2\end{array}$ & $\begin{array}{l}783.8 \\
789.9\end{array}$ & $\begin{array}{l}0.314 \\
0.319\end{array}$ & 1.01 \\
\hline $\begin{array}{r}31 \\
122\end{array}$ & $\begin{array}{l}\text { CHF } \\
\text { CHF }\end{array}$ & $\begin{array}{c}W / O \\
H\end{array}$ & $\begin{array}{l}\text { HP } \\
\text { HP }\end{array}$ & $\begin{array}{l}2000 \\
2000\end{array}$ & $\begin{array}{l}1.000 \\
0.978\end{array}$ & $\begin{array}{l}398.9 \\
400 \div 0\end{array}$ & $\begin{array}{l}376.1 \\
377.3\end{array}$ & $\begin{array}{l}695.5 \\
692.1\end{array}$ & $\begin{array}{l}0.496 \\
0.478\end{array}$ & .96 \\
\hline $\begin{array}{r}33 \\
124\end{array}$ & $\begin{array}{l}\text { CHF } \\
\text { CHF }\end{array}$ & $\begin{array}{c}H / O \\
H\end{array}$ & $\begin{array}{l}\text { HP } \\
\text { HP }\end{array}$ & $\begin{array}{l}2000 \\
2000\end{array}$ & $\begin{array}{l}1.969 \\
2.002\end{array}$ & $\begin{array}{l}400.8 \\
400.0\end{array}$ & $\begin{array}{l}378.1 \\
377.3\end{array}$ & $\begin{array}{l}609.6 \\
610.0\end{array}$ & $\begin{array}{l}0.716 \\
0.723\end{array}$ & 1.01 \\
\hline
\end{tabular}

* W/O AP ans without hot pateh. W IP mans with hot patch.

* Rurs not comparable. 
Comparison of Data With and Without A Hot Patch $-1,5$ Heat Flux Ratio

\begin{tabular}{|c|c|c|c|c|c|c|c|c|c|c|}
\hline $\begin{array}{l}\text { RUN } \\
\text { NO. }\end{array}$ & & TYPE & & $\begin{array}{l}\text { PRES- } \\
\text { (PSIA) }\end{array}$ & $\begin{array}{l}\text { MASS } \\
\text { VELOCITY } \\
X 10-5 \\
\text { ILB/HR- } \\
\text { FISQI }\end{array}$ & $\begin{array}{c}\text { INLET } \\
\text { TEMPER- } \\
\text { ATURE } \\
\text { OEG.F.I }\end{array}$ & $\begin{array}{l}\text { MEASURED } \\
\text { INLET } \\
\text { ENTHALPY } \\
\text { (BTU/LB) }\end{array}$ & $\begin{array}{l}\text { CALCU- } \\
\text { LATED } \\
\text { EXIT } \\
\text { ENTHALPY } \\
\text { (BTU/LB) }\end{array}$ & $\begin{array}{l}\text { A UERAGE } \\
\text { HEAT FLUX } \\
\times 10-6 \\
\text { (ETU/HR- } \\
\text { FTSQ) }\end{array}$ & $\begin{array}{l}\text { RATIO } \\
\text { OF } \\
\text { CRITICAL } \\
\text { HEAT } \\
\text { FLUXES }\end{array}$ \\
\hline $\begin{array}{r}25 \\
101\end{array}$ & $\begin{array}{l}\text { CHF } \\
\text { CHF }\end{array}$ & H/O & $\begin{array}{l}\text { HP } \\
\text { HP }\end{array}$ & $\begin{array}{l}2003 \\
2003\end{array}$ & $\begin{array}{l}0.244 \\
0.245\end{array}$ & $\begin{array}{l}501.4 \\
499.2\end{array}$ & $\begin{array}{r}489.3 \\
486.9\end{array}$ & $\begin{array}{l}885.7 \\
914.1\end{array}$ & $\begin{array}{l}0.150 \\
0.163\end{array}$ & 1.08 \\
\hline $\begin{array}{r}23 \\
108\end{array}$ & $\begin{array}{l}\text { CHF } \\
\text { CHF }\end{array}$ & $\begin{array}{c}H / O \\
H\end{array}$ & $\begin{array}{l}\text { HP } \\
\text { HP }\end{array}$ & $\begin{array}{l}2003 \\
2003\end{array}$ & $\begin{array}{l}0.498 \\
0.502\end{array}$ & $\begin{array}{r}499.7 \\
499.6\end{array}$ & $\begin{array}{l}487.4 \\
487.3\end{array}$ & $\begin{array}{l}800.1 \\
808.4\end{array}$ & $\begin{array}{l}0.242 \\
0.250\end{array}$ & 1.04 \\
\hline $\begin{array}{r}21 \\
114\end{array}$ & $\begin{array}{l}\text { CHF } \\
\text { CHF }\end{array}$ & $\begin{array}{c}W / 0 \\
W\end{array}$ & $\begin{array}{l}\text { HP } \\
\text { HP }\end{array}$ & $\begin{array}{l}2000 \\
2000\end{array}$ & $\begin{array}{l}1.000 \\
0.984\end{array}$ & $\begin{array}{l}500.4 \\
49 \subseteq .2\end{array}$ & $\begin{array}{l}488.2 \\
486.9\end{array}$ & $\begin{array}{l}747.3 \\
740.6\end{array}$ & $\begin{array}{l}0.402 \\
0.388\end{array}$ & .96 \\
\hline $\begin{array}{r}19 \\
132\end{array}$ & $\begin{array}{l}\text { CHF } \\
\text { CHF }\end{array}$ & H/O & $\begin{array}{l}\text { HP } \\
\text { HP }\end{array}$ & $\begin{array}{l}2000 \\
2000\end{array}$ & $\begin{array}{l}1.990 \\
2.002\end{array}$ & $\begin{array}{l}500.2 \\
498.6\end{array}$ & $\begin{array}{l}488.0 \\
486.2\end{array}$ & $\begin{array}{l}690.2 \\
678.4\end{array}$ & $\begin{array}{l}0.625 \\
0.598\end{array}$ & .96 \\
\hline $\begin{array}{r}18 \\
134\end{array}$ & $\begin{array}{l}\text { CHF } \\
\text { CHF }\end{array}$ & $\begin{array}{c}M / 0 \\
n\end{array}$ & $\begin{array}{l}\text { HP } \\
\text { HP }\end{array}$ & $\begin{array}{l}2000 \\
2000\end{array}$ & $\begin{array}{l}2.682 \\
2.626\end{array}$ & $\begin{array}{l}501.1 \\
499.7\end{array}$ & $\begin{array}{l}489.0 \\
487.4\end{array}$ & $\begin{array}{l}660.1 \\
650.6\end{array}$ & $\begin{array}{l}0.713 \\
0.716\end{array}$ & 1.00 \\
\hline $\begin{array}{r}8 \\
144\end{array}$ & $\begin{array}{l}\mathrm{CHF} \\
\mathrm{CHF}\end{array}$ & $\begin{array}{c}H / 0 \\
H\end{array}$ & $\begin{array}{l}\text { HP } \\
\text { HP }\end{array}$ & $\begin{array}{l}2000 \\
2000\end{array}$ & $\begin{array}{l}0.246 \\
0.254\end{array}$ & $\begin{array}{l}596.2 \\
600.1\end{array}$ & $\begin{array}{l}609.3 \\
615.2\end{array}$ & $\begin{array}{l}916.0 \\
940.3\end{array}$ & $\begin{array}{l}0.117 \\
0.128\end{array}$ & 1.10 \\
\hline $\begin{array}{r}10 \\
146\end{array}$ & $\begin{array}{l}\text { CHF } \\
\text { CHF }\end{array}$ & W/O & $\begin{array}{l}\text { HP } \\
\text { HP }\end{array}$ & $\begin{array}{l}2000 \\
2008\end{array}$ & $\begin{array}{l}0.493 \\
0.500\end{array}$ & $\begin{array}{l}599.7 \\
599.7\end{array}$ & $\begin{array}{l}614.6 \\
614.5\end{array}$ & $\begin{array}{l}822.8 \\
831.4\end{array}$ & $\begin{array}{l}0.159 \\
0.168\end{array}$ & 1.06 \\
\hline $\begin{array}{r}12 \\
148\end{array}$ & $\begin{array}{l}\text { CHF } \\
\text { CHF }\end{array}$ & $\begin{array}{c}\text { W/O } \\
4\end{array}$ & $\begin{array}{l}\text { HP } \\
\text { HP }\end{array}$ & $\begin{array}{l}2000 \\
2000\end{array}$ & $\begin{array}{l}1.013 \\
1.020\end{array}$ & $\begin{array}{l}599.9 \\
600.7\end{array}$ & $\begin{array}{l}614.8 \\
616.0\end{array}$ & $\begin{array}{l}772.5 \\
770.3\end{array}$ & $\begin{array}{l}0.248 \\
0.244\end{array}$ & . 98 \\
\hline $\begin{array}{r}14 \\
150\end{array}$ & $\begin{array}{l}\text { CHF } \\
\text { CHF }\end{array}$ & $\begin{array}{c}W / 0 \\
4\end{array}$ & $\begin{array}{l}\text { HP } \\
\text { HP }\end{array}$ & $\begin{array}{l}2000 \\
2000\end{array}$ & $\begin{array}{l}1.986 \\
1.995 \\
.\end{array}$ & $\begin{array}{l}599.4 \\
599.4\end{array}$ & $\begin{array}{l}614.1 \\
614.1\end{array}$ & $\begin{array}{l}738.6 \\
735.8\end{array}$ & $\begin{array}{l}0.384 \\
0.377\end{array}$ & .98 \\
\hline $\begin{array}{r}16 \\
152\end{array}$ & $\begin{array}{l}\text { CHF } \\
\text { CHF }\end{array}$ & $\begin{array}{c}W / 0 \\
H\end{array}$ & $\begin{array}{l}\text { HP } \\
\text { HP }\end{array}$ & $\begin{array}{l}2000 \\
2000\end{array}$ & $\begin{array}{l}2.556 \\
2.754\end{array}$ & $\begin{array}{l}600.2 \\
599.5\end{array}$ & $\begin{array}{l}615.3 \\
614.2\end{array}$ & $\begin{array}{l}723.4 \\
716.8\end{array}$ & $\begin{array}{l}0.429 \\
0.439\end{array}$ & 1.02 \\
\hline
\end{tabular}


TABLE 1 (Continued)

Comparison of Data With and Without A Hot Patch -1.5 Heat Flux Ratio

\begin{tabular}{|c|c|c|c|c|c|c|c|c|c|c|}
\hline $\begin{array}{l}\text { RUN } \\
\text { NO. }\end{array}$ & & TYPE & & $\begin{array}{l}\text { PRES- } \\
\text { (PSIA) }\end{array}$ & $\begin{array}{l}\text { MASS } \\
V E L O C I T Y \\
X 10-6 \\
\text { (LB/HQ- } \\
F Y S O I\end{array}$ & $\begin{array}{c}\text { INLET } \\
\text { TEMPER- } \\
\text { ATURE } \\
\text { (CEG•F•) }\end{array}$ & $\begin{array}{l}\text { MEASURED } \\
\text { INLET } \\
\text { ENTHALPY } \\
\text { IBTU/L BI }\end{array}$ & $\begin{array}{l}\text { CALCU- } \\
\text { LATED } \\
\text { EXIT } \\
\text { ENTHALPY } \\
\text { (BTU/LB) }\end{array}$ & $\begin{array}{l}\text { A VERAGE } \\
\text { HEAT FLUX } \\
\text { X 10-6 } \\
\text { (BTU/HR- } \\
\text { FTSQ) }\end{array}$ & $\begin{array}{l}\text { RATIO } \\
\text { OF } \\
\text { CRITICAL } \\
\text { HEAT } \\
\text { FLUXES }\end{array}$ \\
\hline $\begin{array}{l}71 \\
90\end{array}$ & $\begin{array}{l}\text { CHF } \\
\text { CHF }\end{array}$ & $\begin{array}{c}W / O \\
W\end{array}$ & $\begin{array}{l}\text { HP } \\
\text { HP }\end{array}$ & $\begin{array}{l}1600 \\
1600 .\end{array}$ & $\begin{array}{l}0.250 \\
0.252\end{array}$ & $\begin{array}{l}402.5 \\
398.8\end{array}$ & $\begin{array}{l}379.5 \\
375.6\end{array}$ & $\begin{array}{l}842.5 \\
881.2\end{array}$ & $\begin{array}{l}0.180 \\
0.198\end{array}$ & 1.10 \\
\hline $\begin{array}{l}73 \\
92\end{array}$ & $\begin{array}{l}\text { CHF } \\
\text { CHF }\end{array}$ & $\begin{array}{c}H / O \\
H\end{array}$ & $\begin{array}{l}\text { HP } \\
\text { HP }\end{array}$ & $\begin{array}{l}1600 \\
1600\end{array}$ & $\begin{array}{l}0.501 \\
0.502\end{array}$ & $\begin{array}{l}401.3 \\
400.2\end{array}$ & $\begin{array}{l}378.3 \\
377.1\end{array}$ & $\begin{array}{l}728.3 \\
757.0\end{array}$ & $\begin{array}{l}0.272 \\
0.296\end{array}$ & 1.09 \\
\hline $\begin{array}{r}75 \\
125\end{array}$ & $\begin{array}{l}\text { CHF } \\
\text { CHF }\end{array}$ & $\begin{array}{c}H / O \\
H\end{array}$ & $\begin{array}{l}\text { MP } \\
\text { HP }\end{array}$ & $\begin{array}{l}1600 \\
1600\end{array}$ & $\begin{array}{l}1.010 \\
0.988\end{array}$ & $\begin{array}{l}400.6 \\
400.1\end{array}$ & $\begin{array}{l}377.5 \\
377.0\end{array}$ & $\begin{array}{l}681.8 \\
377.0\end{array}$ & $\begin{array}{r}0.478 \\
\ldots-.0\end{array}$ & 0. \\
\hline $\begin{array}{r}77 \\
131\end{array}$ & $\begin{array}{l}\text { CHF } \\
\text { CHF }\end{array}$ & $\begin{array}{c}H / O \\
H\end{array}$ & $\begin{array}{l}\text { HP } \\
\text { HP }\end{array}$ & $\begin{array}{l}1600 \\
1600\end{array}$ & $\begin{array}{l}2.003 \\
2.030\end{array}$ & $\begin{array}{l}401.0 \\
400.1\end{array}$ & $\begin{array}{l}378.0 \\
377.0\end{array}$ & $\begin{array}{l}610.4 \\
607.2\end{array}$ & $\begin{array}{l}0.723 \\
0.726\end{array}$ & 1.00 \\
\hline $\begin{array}{r}69 \\
103\end{array}$ & $\begin{array}{l}\text { CHF } \\
\text { CHF }\end{array}$ & $\begin{array}{c}W / O \\
H\end{array}$ & $\begin{array}{l}\text { HP } \\
\text { HP }\end{array}$ & $\begin{array}{l}1600 \\
1600\end{array}$ & $\begin{array}{l}0.250 \\
0.254\end{array}$ & $\begin{array}{l}499.3 \\
499.7\end{array}$ & $\begin{array}{l}486.9 \\
487.3\end{array}$ & $\begin{array}{l}868.4 \\
901.6\end{array}$ & $\begin{array}{l}0.148 \\
0.163\end{array}$ & 1.10 \\
\hline $\begin{array}{r}67 \\
110\end{array}$ & $\begin{array}{l}\mathrm{CHF} \\
\mathrm{CHF}\end{array}$ & $H / O$ & $\begin{array}{l}\text { HP } \\
\text { HP }\end{array}$ & $\begin{array}{l}1600 \\
1600\end{array}$ & $\begin{array}{l}0.508 \\
0.500\end{array}$ & $\begin{array}{l}499.7 \\
498.8\end{array}$ & $\begin{array}{l}487.4 \\
486.3\end{array}$ & $\begin{array}{l}763.4 \\
778.9\end{array}$ & $\begin{array}{l}0.218 \\
0.227\end{array}$ & 1.04 \\
\hline $\begin{array}{r}65 \\
116\end{array}$ & $\begin{array}{l}\text { CHF } \\
\text { CHF }\end{array}$ & $W / O$ & $\begin{array}{l}\text { HP } \\
\text { HP }\end{array}$ & $\begin{array}{l}1600 \\
1600\end{array}$ & $\begin{array}{l}0.997 \\
1.029\end{array}$ & $\begin{array}{l}499.7 \\
498.8\end{array}$ & $\begin{array}{l}487.3 \\
486.3\end{array}$ & $\begin{array}{l}708.2 \\
709.6\end{array}$ & $\begin{array}{l}0.342 \\
0.357\end{array}$ & 1.04 \\
\hline $\begin{array}{r}63 \\
136\end{array}$ & $\begin{array}{l}\text { CHF } \\
\text { CHF }\end{array}$ & ${ }_{H}^{H} O$ & $\begin{array}{l}\text { HP } \\
\text { HP }\end{array}$ & $\begin{array}{l}1600 \\
1600\end{array}$ & $\begin{array}{l}1.989 \\
2.001\end{array}$ & $\begin{array}{l}500.1 \\
499.4\end{array}$ & $\begin{array}{l}487.8 \\
487.0\end{array}$ & $\begin{array}{l}675.6 \\
670.3\end{array}$ & $\begin{array}{l}0.580 \\
0.570\end{array}$ & .98 \\
\hline $\begin{array}{r}61 \\
142\end{array}$ & $\begin{array}{l}\text { NO } 5 \\
\text { CHF }\end{array}$ & DATA & HP & 1600 & 2.852 & 499.0 & 486.6 & 642.2 & 0.689 & 0. \\
\hline
\end{tabular}


TABLE 1 (Continued)

Comparison of Data With and Without A Hot Patch -1.5 Heat Flux Ratio

\begin{tabular}{|c|c|c|c|c|c|c|c|c|c|c|}
\hline $\begin{array}{l}\text { RUN } \\
\text { NO. }\end{array}$ & & TYPE & . & $\begin{array}{l}\text { गRES - } \\
\text { (DSIA) }\end{array}$ & $\begin{array}{l}\text { MASS } \\
\text { VELOCITY } \\
\times 1 O-E \\
\text { TLB/HR- } \\
\text { F(SO) }\end{array}$ & $\begin{array}{l}\text { INLET } \\
\text { TEMPER- } \\
\text { ATURE } \\
\text { (OEG.F.) }\end{array}$ & $\begin{array}{l}\text { MEASURED } \\
\text { INLET } \\
\text { ENTHALPY } \\
\text { (BTU/LB) }\end{array}$ & $\begin{array}{l}\text { CALCU- } \\
\text { LATEO } \\
\text { EXIT } \\
\text { ENTHALP } \\
\text { (BTUILB: }\end{array}$ & $\begin{array}{l}\text { A VERAGE } \\
\text { HEAT FLUX } \\
\times 10-6 \\
\text { (BTU/HR- } \\
\text { FTSQ) }\end{array}$ & $\begin{array}{l}\text { RATIO } \\
\text { OF } \\
\text { CRITICAL } \\
\text { HEAT } \\
\text { FLUXES }\end{array}$ \\
\hline $\begin{array}{r}40 \\
154\end{array}$ & $\begin{array}{l}\text { CHF } \\
\text { CHF }\end{array}$ & $\begin{array}{c}H / O \\
H\end{array}$ & $\begin{array}{l}H P \\
H P\end{array}$ & $\begin{array}{l}1200 \\
1200\end{array}$ & $\begin{array}{l}1.245 \\
0.253\end{array}$ & $\begin{array}{l}200 \cdot 2 \\
200.0\end{array}$ & $\begin{array}{l}171.1 \\
170.9\end{array}$ & $\begin{array}{l}704.3 \\
820.1\end{array}$ & $\begin{array}{l}B .203 \\
0.255\end{array}$ & 1.26 \\
\hline $\begin{array}{r}42 \\
156\end{array}$ & $\begin{array}{l}\text { CHF } \\
\text { CHF }\end{array}$ & $\begin{array}{c}\omega / O \\
H\end{array}$ & $\begin{array}{l}\text { HP } \\
\text { HP }\end{array}$ & $\begin{array}{l}1200 \\
1200\end{array}$ & $\begin{array}{l}0.501 \\
0.501\end{array}$ & $\begin{array}{l}199.8 \\
200.4\end{array}$ & $\begin{array}{l}170.6 \\
171.2\end{array}$ & $\begin{array}{l}689.0 \\
698.2\end{array}$ & $\begin{array}{l}0.404 \\
0.410\end{array}$ & 1.02 \\
\hline $\begin{array}{l}44 \\
88\end{array}$ & $\begin{array}{l}\text { CHF } \\
\text { CHF }\end{array}$ & $\begin{array}{c}H / O \\
H\end{array}$ & $\begin{array}{l}\text { HP } \\
\text { HP }\end{array}$ & $\begin{array}{l}1200 \\
1200\end{array}$ & $\begin{array}{l}0.247 \\
0.257\end{array}$ & $\begin{array}{l}401.0 \\
399.5\end{array}$ & $\begin{array}{l}377.5 \\
375.9\end{array}$ & $\begin{array}{l}814.3 \\
866.0\end{array}$ & $\begin{array}{l}0.167 \\
0.196\end{array}$ & 1.17 \\
\hline $\begin{array}{l}46 \\
94\end{array}$ & $\begin{array}{l}\text { CHF } \\
\text { CHF }\end{array}$ & $\begin{array}{c}H / 0 \\
n\end{array}$ & $\begin{array}{l}\text { HP } \\
\text { HP }\end{array}$ & $\begin{array}{l}1200 \\
1200\end{array}$ & $\begin{array}{l}0.493 \\
0.500\end{array}$ & $\begin{array}{l}399.8 \\
400.2\end{array}$ & $\begin{array}{l}376.2 \\
376.7\end{array}$ & $\begin{array}{l}735.5 \\
731.3\end{array}$ & $\begin{array}{l}0.275 \\
0.276\end{array}$ & 1.00 \\
\hline $\begin{array}{c}48 \\
128^{*}\end{array}$ & $\begin{array}{l}\text { CHF } \\
\text { NO } 0\end{array}$ & $\begin{array}{r}\text { H/O } \\
\text { OATA }\end{array}$ & HP & 1200 & 0.921 & 400.5 & 377.0 & 681.4 & 0.435 & \\
\hline $\begin{array}{r}48 A \\
12 B *\end{array}$ & $\begin{array}{l}\text { CHF } \\
\text { NO } D\end{array}$ & $\begin{array}{l}\text { H/O } \\
\text { DATA }\end{array}$ & HP & $\therefore 200$ & B. 987 & 400.7 & 377.2 & 684.6 & 0.471 & Repeat \\
\hline $\begin{array}{r}50 \\
130\end{array}$ & $\begin{array}{l}\text { CHF } \\
\text { CHF }\end{array}$ & $\begin{array}{c}H>0 \\
H\end{array}$ & $\begin{array}{l}\text { HP } \\
\text { HP }\end{array}$ & $\begin{array}{l}1200 \\
1200\end{array}$ & $\begin{array}{l}2.010 \\
1.973\end{array}$ & $\begin{array}{l}400.9 \\
399.4\end{array}$ & $\begin{array}{l}377 \cdot 4 \\
375.8\end{array}$ & $\begin{array}{l}609.6 \\
614.6\end{array}$ & $\begin{array}{l}0.725 \\
0.732\end{array}$ & 1.01 \\
\hline $\begin{array}{r}51 \\
105\end{array}$ & $\begin{array}{l}\text { CHF } \\
\text { CHF }\end{array}$ & $\begin{array}{c}H / 0 \\
H\end{array}$ & $\begin{array}{l}\text { HP } \\
\text { HP }\end{array}$ & $\begin{array}{l}1200 \\
=200\end{array}$ & $\begin{array}{l}3.248 \\
3.251\end{array}$ & $\begin{array}{l}501.0 \\
500.2\end{array}$ & $\begin{array}{l}489.0 \\
488.2\end{array}$ & $\begin{array}{l}837.4 \\
887.4\end{array}$ & $\begin{array}{l}0.134 \\
0.155\end{array}$ & 1.16 \\
\hline $\begin{array}{r}53 \\
112\end{array}$ & $\begin{array}{l}\text { CHF } \\
\text { CHF }\end{array}$ & $\begin{array}{c}\mathrm{H} / \mathrm{O} \\
\mathrm{H}\end{array}$ & $\begin{array}{l}\text { HP } \\
\text { HP }\end{array}$ & $\begin{array}{l}1200 \\
-200\end{array}$ & $\begin{array}{l}0.471 \\
3.498\end{array}$ & $\begin{array}{l}500.7 \\
500.1\end{array}$ & $\begin{array}{l}488.6 \\
488.0\end{array}$ & $\begin{array}{l}756.2 \\
761.4\end{array}$ & $\begin{array}{l}0.196 \\
0.212\end{array}$ & 1.08 \\
\hline $\begin{array}{r}55 \\
118\end{array}$ & $\begin{array}{l}\text { CHF } \\
\text { CHF }\end{array}$ & $\begin{array}{c}W / 0 \\
u\end{array}$ & $\begin{array}{l}\text { HP } \\
\text { HP }\end{array}$ & $\begin{array}{l}=200 \\
-200\end{array}$ & $\begin{array}{l}1.093 \\
0.980\end{array}$ & $\begin{array}{l}500.2 \\
499.0\end{array}$ & $\begin{array}{l}488.0 \\
486.7\end{array}$ & $\begin{array}{l}723.13 \\
704.7\end{array}$ & $\begin{array}{l}0.366 \\
0.332\end{array}$ & .91 \\
\hline $\begin{array}{r}57 \\
138\end{array}$ & $\begin{array}{l}\text { CHF } \\
\text { CHF }\end{array}$ & $\begin{array}{c}M / O \\
H\end{array}$ & $\begin{array}{l}\text { HP } \\
\text { HP }\end{array}$ & $\begin{array}{l}1200 \\
1200\end{array}$ & $\begin{array}{l}2.014 \\
2.003\end{array}$ & $\begin{array}{l}500.2 \\
500.1\end{array}$ & $\begin{array}{l}488.1 \\
488.0\end{array}$ & $\begin{array}{l}667.5 \\
655.0\end{array}$ & $\begin{array}{l}0.562 \\
0.520\end{array}$ & .93 \\
\hline
\end{tabular}

* No data taken at CFF leve1s, see Run 129 at 98 CHF levels. 
TABLE 1 (Continued)

Comparison of Data With and Without A Hot Patch -1.5 Heat Flux Ratio

\begin{tabular}{|c|c|c|c|c|c|c|c|c|c|c|}
\hline $\begin{array}{l}\text { RUN } \\
\text { NO. }\end{array}$ & $\star$ & YPE & & $\begin{array}{l}\text { PRES- } \\
\text { (PSIA) }\end{array}$ & $\begin{array}{l}\text { MASS } \\
\text { VELOCITY } \\
\text { X } 1 \text { CS-6 } \\
\text { (LB/HR- } \\
\text { FTSQ) }\end{array}$ & $\begin{array}{c}\text { INLET } \\
\text { TEMPER- } \\
\text { ATURE } \\
\text { (DEG.F•) }\end{array}$ & $\begin{array}{l}\text { MEASURED } \\
\text { INLET } \\
\text { ENTHALPY } \\
\text { (BTU/LB) }\end{array}$ & $\begin{array}{l}\text { CALCU - } \\
\text { LATEO } \\
\text { EXIT } \\
\text { ENTHALPY } \\
\text { (BTU/LB) }\end{array}$ & $\begin{array}{l}\text { A VERAGE } \\
\text { HEAT FLUX } \\
\text { X } 10-6 \\
\text { (BTU/HR- } \\
\text { FTSQ) }\end{array}$ & $\begin{array}{l}\text { Ratio of } \\
\text { HEAT } \\
\text { FLU XES }\end{array}$ \\
\hline $\begin{array}{l}39 \\
83\end{array}$ & $\begin{array}{l}98 \\
98\end{array}$ & $\begin{array}{c}W / 0 \\
H\end{array}$ & $\begin{array}{l}\text { HP } \\
\text { HP }\end{array}$ & $\begin{array}{l}2000 \\
2000 .\end{array}$ & $\begin{array}{l}0.258 \\
0.253\end{array}$ & $\begin{array}{r}201.6 \\
199.7\end{array}$ & $\begin{array}{l}174.3 \\
172.4\end{array}$ & $\begin{array}{l}769.8 \\
844.6\end{array}$ & $\begin{array}{l}0.239 \\
0.265\end{array}$ & 1.11 \\
\hline 37 & 98 & $w / 0$ & HP & 2000 & 0.498 & 201.6 & 174.3 & 695.8 & 0.403 & \\
\hline $\begin{array}{r}35 \\
159\end{array}$ & $\begin{array}{l}98 \\
98\end{array}$ & $\begin{array}{c}M / O \\
H\end{array}$ & $\begin{array}{l}\text { HP } \\
\text { HP }\end{array}$ & $\begin{array}{l}2000 \\
2000\end{array}$ & $\begin{array}{l}0.991 \\
1.010\end{array}$ & $\begin{array}{l}200.7 \\
427.8\end{array}$ & $\begin{array}{l}173.4 \\
407.4\end{array}$ & $\begin{array}{l}625.2 \\
699.4\end{array}$ & $\begin{array}{l}0.695 \\
0.458\end{array}$ & ** \\
\hline $\begin{array}{l}28 \\
86\end{array}$ & $\begin{array}{l}98 \\
98\end{array}$ & $\begin{array}{c}W / O \\
W\end{array}$ & $\begin{array}{l}\text { HP } \\
\text { HP }\end{array}$ & $\begin{array}{l}2000 \\
2000\end{array}$ & $\begin{array}{l}0.244 \\
0: 254\end{array}$ & $\begin{array}{l}401.5 \\
400.7\end{array}$ & $\begin{array}{l}378.9 \\
378.0\end{array}$ & $\begin{array}{l}848.5 \\
878.2\end{array}$ & $\begin{array}{l}0.178 \\
0.197\end{array}$ & 1.11 \\
\hline $\begin{array}{r}30 \\
100\end{array}$ & $\begin{array}{l}98 \\
98\end{array}$ & $\begin{array}{c}H / O \\
H\end{array}$ & $\begin{array}{l}\text { HP } \\
\text { HP }\end{array}$ & $\begin{array}{l}2000 \\
2000\end{array}$ & $\begin{array}{l}0.500 \\
0.495\end{array}$ & $\begin{array}{l}402.2 \\
400.1\end{array}$ & $\begin{array}{l}379.7 \\
377.4\end{array}$ & $\begin{array}{l}780.2 \\
781.5\end{array}$ & $\begin{array}{l}0.311 \\
0.311\end{array}$ & 1.00 \\
\hline $\begin{array}{r}32 \\
123\end{array}$ & $\begin{array}{l}98 \\
98\end{array}$ & $\begin{array}{c}H / O \\
H\end{array}$ & $\begin{array}{l}\text { HP } \\
\text { HP }\end{array}$ & $\begin{array}{l}2000 \\
2000\end{array}$ & $\begin{array}{l}1.0 C 0 \\
0.985\end{array}$ & $\begin{array}{l}399.0 \\
400.0\end{array}$ & $\begin{array}{l}376.2 \\
377.3\end{array}$ & $\begin{array}{l}691.8 \\
687.2\end{array}$ & $\begin{array}{l}0.490 \\
0.474\end{array}$ & .97 \\
\hline
\end{tabular}

* Type 98 H/0 EP ans a test run at $98 \%$ of CHP without hot patch.

* Runs not comparable. 
TABLE 1 (Continued)

Comparison of Data With and Without A Hot Patch -1.5 Heat Flux Ratio

\begin{tabular}{|c|c|c|c|c|c|c|c|c|c|c|}
\hline $\begin{array}{l}\text { RUN } \\
\text { NO. }\end{array}$ & & TYPE & & $\begin{array}{l}\text { PRES- } \\
\text { (PSIA) }\end{array}$ & $\begin{array}{l}\text { MASS } \\
\text { VELOCITY } \\
\times \text { IO-6 } \\
\text { (LB/HR- } \\
\text { FISO) }\end{array}$ & $\begin{array}{l}\text { INLET } \\
\text { TEMPER- } \\
\text { ATURE } \\
\text { (DEG.F.) }\end{array}$ & $\begin{array}{l}\text { MEASURED } \\
\text { INLET } \\
\text { ENTHALPY } \\
\text { (BTU/LLB) }\end{array}$ & $\begin{array}{l}\text { CALCU - } \\
\text { LATED } \\
\text { EXIT } \\
\text { ENTHALPY } \\
\text { (BTU/LB) }\end{array}$ & $\begin{array}{l}\text { AVERAGE } \\
\text { HEAT FLUX } \\
\times 10-6 \\
\text { (BTU/HR- } \\
\text { FTSQ) }\end{array}$ & $\begin{array}{l}\text { Ratio of } \\
\text { HEAT } \\
\text { FLUXES }\end{array}$ \\
\hline $\begin{array}{r}26 \\
102\end{array}$ & $\begin{array}{l}98 \\
98\end{array}$ & $\begin{array}{c}H / O \\
H\end{array}$ & $\begin{array}{l}\text { MP } \\
\text { HP }\end{array}$ & $\begin{array}{l}2000 \\
2000\end{array}$ & $\begin{array}{l}0.243 \\
0.246\end{array}$ & $\begin{array}{l}502.0 \\
499.0\end{array}$ & $\begin{array}{r}490.1 \\
-486.6\end{array}$ & $\begin{array}{l}877.1 \\
901.8\end{array}$ & $\begin{array}{l}0.146 \\
0.158\end{array}$ & 1.08 \\
\hline $\begin{array}{r}24 \\
109\end{array}$ & $\begin{array}{l}98 \\
98\end{array}$ & $\begin{array}{c}M / O \\
H\end{array}$ & $\begin{array}{l}\text { HP } \\
\text { HP }\end{array}$ & $\begin{array}{l}2000 \\
2000\end{array}$ & $\begin{array}{l}0.495 \\
0.502\end{array}$ & $\begin{array}{r}499.6 \\
499.6\end{array}$ & $\begin{array}{l}487.3 \\
487.3\end{array}$ & $\begin{array}{l}794.8 \\
801.3\end{array}$ & $\begin{array}{l}0.237 \\
0.245\end{array}$ & 1.03 \\
\hline $\begin{array}{r}22 \\
115\end{array}$ & $\begin{array}{l}98 \\
98\end{array}$ & $\begin{array}{c}H / O \\
H\end{array}$ & $\begin{array}{l}\text { HP } \\
\text { HP }\end{array}$ & $\begin{array}{l}2000 \\
2000\end{array}$ & $\begin{array}{l}1.000 \\
0.384\end{array}$ & $\begin{array}{l}500.6 \\
499.0\end{array}$ & $\begin{array}{l}488.5 \\
486.7\end{array}$ & $\begin{array}{l}742.8 \\
736.5\end{array}$ & $\begin{array}{l}0.395 \\
0.382\end{array}$ & .97 \\
\hline $\begin{array}{r}20 \\
133\end{array}$ & $\begin{array}{l}98 \\
98\end{array}$ & $\begin{array}{c}H / O \\
H\end{array}$ & $\begin{array}{l}\text { HP } \\
\text { HP }\end{array}$ & $\begin{array}{l}2000 \\
2000\end{array}$ & $\begin{array}{l}1.997 \\
2.000\end{array}$ & $\begin{array}{r}499.6 \\
498.5\end{array}$ & $\begin{array}{l}487.4 \\
486.0\end{array}$ & $\begin{array}{l}68.8 .8 \\
674.7\end{array}$ & $\begin{array}{l}0.612 \\
0.586\end{array}$ & .96 \\
\hline $\begin{array}{r}59 * \\
135\end{array}$ & $\begin{array}{l}\text { NO } \\
98\end{array}$ & $\begin{array}{c}\text { DATA } \\
W\end{array}$ & HP & 2000 & 2.923 & 499.3 & 487.0 & 648.5 & 0.708 & 0 \\
\hline $\begin{array}{r}9 \\
145\end{array}$ & $\begin{array}{l}98 \\
98\end{array}$ & $\begin{array}{c}H / O \\
H\end{array}$ & $\begin{array}{l}\text { HP } \\
\text { HP }\end{array}$ & $\begin{array}{l}2000 \\
2000\end{array}$ & $\begin{array}{l}0.248 \\
0.255\end{array}$ & $\begin{array}{l}596.1 \\
600.0\end{array}$ & $\begin{array}{l}609.3 \\
614.9\end{array}$ & $\begin{array}{l}904.9 \\
929.5\end{array}$ & $\begin{array}{l}0.114 \\
0.125\end{array}$ & 1.09 \\
\hline $\begin{array}{r}11 \\
147\end{array}$ & $\begin{array}{l}98 \\
98\end{array}$ & $\begin{array}{c}W / 0 \\
W\end{array}$ & $\begin{array}{l}\text { HP } \\
\text { HP }\end{array}$ & $\begin{array}{l}2000 \\
2000\end{array}$ & $\begin{array}{l}0.492 \\
0.505\end{array}$ & $\begin{array}{l}599.6 \\
599.4\end{array}$ & $\begin{array}{l}614.4 \\
614.1\end{array}$ & $\begin{array}{l}818.9 \\
823.6\end{array}$ & $\begin{array}{l}0.156 \\
0.164\end{array}$ & 1.05 \\
\hline $\begin{array}{r}13 \\
149\end{array}$ & $\begin{array}{l}98 \\
98\end{array}$ & $\begin{array}{c}W / O \\
H\end{array}$ & $\begin{array}{l}\text { HP } \\
\text { HP }\end{array}$ & $\begin{array}{l}2000 \\
2000\end{array}$ & $\begin{array}{l}1.013 \\
1.019\end{array}$ & $\begin{array}{l}599.5 \\
600.6\end{array}$ & $\begin{array}{l}614.2 \\
615.8\end{array}$ & $\begin{array}{l}768.6 \\
767.1\end{array}$ & $\begin{array}{l}0.243 \\
0.239\end{array}$ & .99 \\
\hline $\begin{array}{r}15 \\
151\end{array}$ & $\begin{array}{l}98 \\
98\end{array}$ & H/O & $\begin{array}{l}\text { HP } \\
\text { HP }\end{array}$ & $\begin{array}{l}2000 \\
2000\end{array}$ & $\begin{array}{l}1.983 \\
1.998\end{array}$ & $\begin{array}{l}599.8 \\
599.3\end{array}$ & $\begin{array}{l}613.5 \\
613.9\end{array}$ & $\begin{array}{l}736.2 \\
731.6\end{array}$ & $\begin{array}{l}0.378 \\
0.365\end{array}$ & .97 \\
\hline $\begin{array}{r}17 \\
153\end{array}$ & $\begin{array}{l}98 \\
98\end{array}$ & $\begin{array}{l}W / 0 \\
H\end{array}$ & $\begin{array}{l}\text { HP } \\
\text { HP }\end{array}$ & $\begin{array}{l}2000 \\
2000\end{array}$ & $\begin{array}{l}2.561 \\
2.758\end{array}$ & $\begin{array}{l}599.5 \\
599.0\end{array}$ & $\begin{array}{l}614 \cdot 3 \\
613 \cdot 4\end{array}$ & $\begin{array}{l}720.1 \\
712.8\end{array}$ & $\begin{array}{l}0.421 \\
0.426\end{array}$ & 1.01 \\
\hline
\end{tabular}

* No data taken at C.FF levels, see Run 60 at $98 \mathrm{CHF}$ levels. 
TABLE 1 (Continued)

Comparison of Data With and Without A Hot Patch -1.5 Heat Flux Ratio

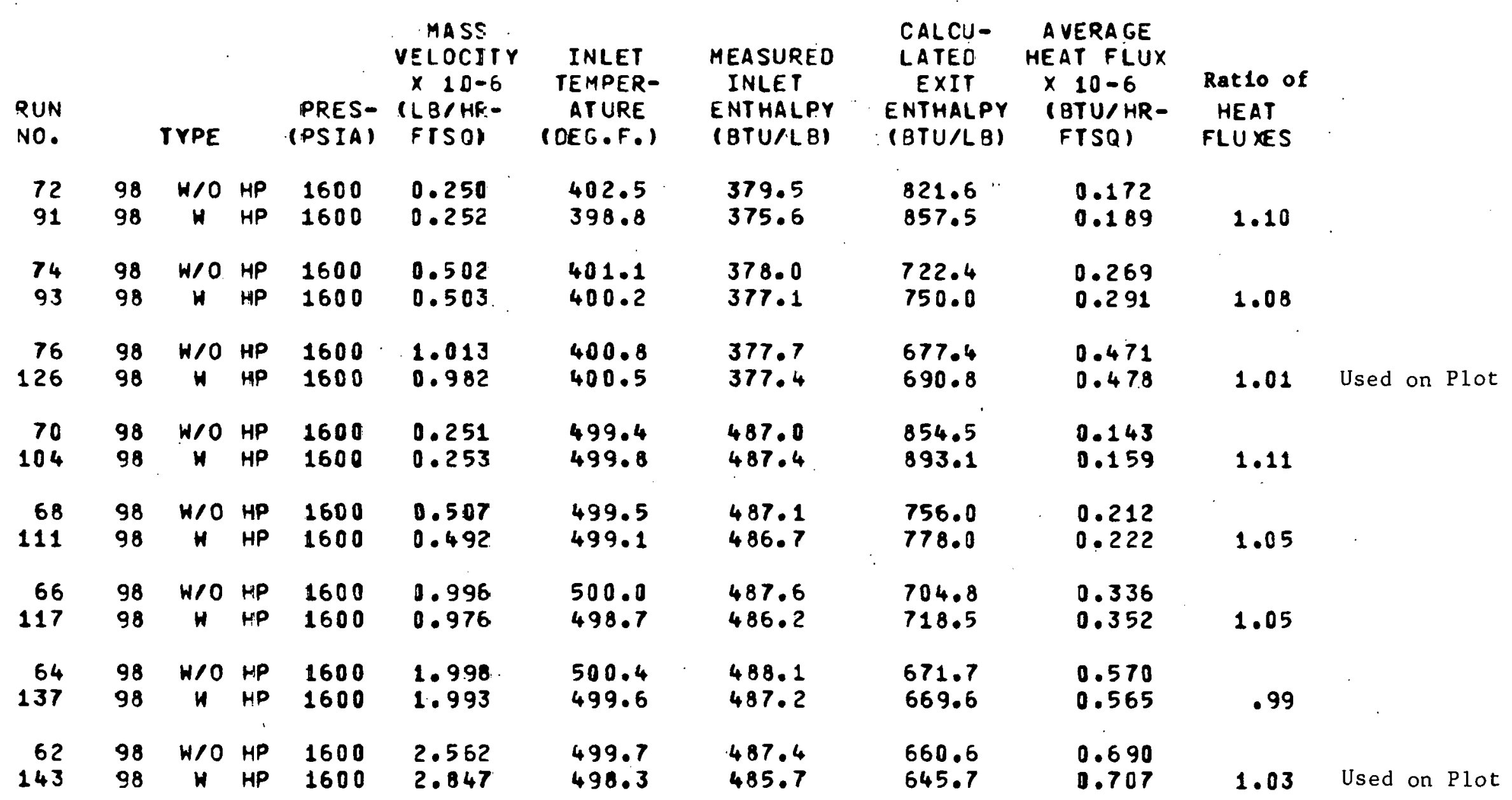


TABIE 1 (Continued)

Comparison of Data With and Without A Hot Patch -1.5 Heat Flux Ratio

\begin{tabular}{|c|c|c|c|c|c|c|c|c|c|c|c|}
\hline $\begin{array}{l}\text { RUN } \\
\text { NO. }\end{array}$ & & TYPE & & $\begin{array}{l}\text { PRES- } \\
\text { (PSIA) }\end{array}$ & $\begin{array}{l}\text { MASS } \\
V E L O C I T Y \\
X 10-6 \\
\text { ILB/HR- } \\
\text { FTSOI }\end{array}$ & $\begin{array}{c}\text { INLET } \\
\text { TEMPER- } \\
\text { ATURE } \\
\text { (DEG.F•) }\end{array}$ & $\begin{array}{l}\text { MEASURED } \\
\text { INLET } \\
\text { ENTHALPY } \\
\text { (BTU/LLB) }\end{array}$ & $\begin{array}{l}\text { CALCU- } \\
\text { LATEO } \\
\text { EXIT } \\
\text { ENTHALPY } \\
\text { (BTU/LB) }\end{array}$ & $\begin{array}{l}\text { A YERAGE } \\
\text { HEAT FLUX } \\
\text { X 10-6 } \\
\text { (BTU/ HR- } \\
\text { FTSQ) }\end{array}$ & $\begin{array}{l}\text { Rat10 of } \\
\text { HEAT } \\
\text { FLUXES }\end{array}$ & \\
\hline $\begin{array}{r}41 \\
155\end{array}$ & $\begin{array}{l}98 \\
98\end{array}$ & $\begin{array}{c}H / O \\
H\end{array}$ & $\begin{array}{l}\text { HP } \\
\text { HP }\end{array}$ & $\begin{array}{l}1200 \\
1200\end{array}$ & $\begin{array}{l}0.245 \\
0.253\end{array}$ & $\begin{array}{l}200.2 \\
200.1\end{array}$ & $\begin{array}{l}171.0 \\
170.9\end{array}$ & $\begin{array}{l}677.3 \\
806.9\end{array}$ & $\begin{array}{l}0.193 \\
0.250\end{array}$ & 1.29 & \\
\hline $\begin{array}{r}43 \\
157\end{array}$ & $\begin{array}{l}98 \\
98\end{array}$ & $\begin{array}{c}W / 0 \\
W\end{array}$ & $\begin{array}{l}\text { HP } \\
\text { HP }\end{array}$ & $\begin{array}{l}1200 \\
1200\end{array}$ & $\begin{array}{l}0.501 \\
0.501\end{array}$ & $\begin{array}{l}200.0 \\
200.2\end{array}$ & $\begin{array}{l}170.8 \\
171.1\end{array}$ & $\begin{array}{l}682.0 \\
686.7\end{array}$ & $\begin{array}{l}0.398 \\
0.401\end{array}$ & 1.01 & \\
\hline $\begin{array}{l}45 \\
89\end{array}$ & $\begin{array}{l}98 \\
98\end{array}$ & $\begin{array}{c}H / O \\
H\end{array}$ & $\begin{array}{l}\text { HP } \\
\text { HP }\end{array}$ & $\begin{array}{l}1200 \\
1200\end{array}$ & $\begin{array}{l}0.246 \\
0.257\end{array}$ & $\begin{array}{l}400.7 \\
399.3\end{array}$ & $\begin{array}{l}377.2 \\
375.7\end{array}$ & $\begin{array}{l}803.5 \\
341.7\end{array}$ & $\begin{array}{l}0.163 \\
0.186\end{array}$ & 1.14 & \\
\hline $\begin{array}{l}47 \\
95\end{array}$ & $\begin{array}{l}98 \\
98\end{array}$ & $H / O$ & $\begin{array}{l}\text { HP } \\
\text { HP }\end{array}$ & $\begin{array}{l}1200 \\
1200\end{array}$ & $\begin{array}{l}1.494 \\
0.499\end{array}$ & $\begin{array}{l}: 99.8 \\
400.3\end{array}$ & $\begin{array}{l}376.3 \\
376.8\end{array}$ & $\begin{array}{l}729.1 \\
723.5\end{array}$ & $\begin{array}{l}0.271 \\
0.269\end{array}$ & •99 & \\
\hline $\begin{array}{r}49 \\
129\end{array}$ & $\begin{array}{l}98 \\
98\end{array}$ & $\begin{array}{c}H / O \\
H\end{array}$ & $\begin{array}{l}\text { MP } \\
\text { HP }\end{array}$ & $\begin{array}{l}1200 \\
1200\end{array}$ & $\begin{array}{l}0.923 \\
0.984\end{array}$ & $\begin{array}{l}401.1 \\
398.9\end{array}$ & $\begin{array}{l}377.7 \\
375.2\end{array}$ & $\begin{array}{l}676.2 \\
673.2\end{array}$ & $\begin{array}{l}0.428 \\
0.455\end{array}$ & 1.06 & \\
\hline $\begin{array}{l}494 \\
129\end{array}$ & $\begin{array}{l}98 \\
98\end{array}$ & $\begin{array}{c}H / O \\
H\end{array}$ & $\begin{array}{l}\text { HP } \\
\text { HP }\end{array}$ & $\begin{array}{l}1200 \\
1200\end{array}$ & $\begin{array}{l}0.989 \\
0.984\end{array}$ & $\begin{array}{l}401.1 \\
398.9\end{array}$ & $\begin{array}{l}377.6 \\
375.2\end{array}$ & $\begin{array}{l}678.5 \\
673.2\end{array}$ & $\begin{array}{l}0.462 \\
0.455\end{array}$ & .99 & Used on Plot \\
\hline $\begin{array}{r}52 \\
106\end{array}$ & $\begin{array}{l}98 \\
98\end{array}$ & $\begin{array}{c}k / 0 \\
H\end{array}$ & $\begin{array}{l}\text { HP } \\
\text { HP }\end{array}$ & $\begin{array}{l}1200 \\
1200\end{array}$ & $\begin{array}{l}0.248 \\
0.251\end{array}$ & $\begin{array}{l}501.6 \\
4919.5\end{array}$ & $\begin{array}{l}489.7 \\
487.3\end{array}$ & $\begin{array}{l}815.3 \\
874.1\end{array}$ & $\begin{array}{l}0.125 \\
0.151\end{array}$ & 1.20 & \\
\hline $\begin{array}{r}54 \\
113\end{array}$ & $\begin{array}{l}98 \\
98\end{array}$ & $\begin{array}{c}\text { W/O } \\
H\end{array}$ & $\begin{array}{l}\text { HP } \\
\text { HP }\end{array}$ & $\begin{array}{l}1200 \\
1200\end{array}$ & $\begin{array}{l}0.471 \\
0.498\end{array}$ & $\begin{array}{l}500.7 \\
500.2\end{array}$ & $\begin{array}{l}488.6 \\
488.0\end{array}$ & $\begin{array}{l}745.1 \\
753.5\end{array}$ & $\begin{array}{l}0.188 \\
0.205\end{array}$ & 1.09 & \\
\hline $\begin{array}{r}56 \\
119\end{array}$ & $\begin{array}{l}98 \\
98\end{array}$ & $\begin{array}{c}W / 0 \\
W\end{array}$ & $\begin{array}{l}\text { HP } \\
\text { HP }\end{array}$ & $\begin{array}{l}1200 \\
1200\end{array}$ & $\begin{array}{l}1.008 \\
0.977\end{array}$ & $\begin{array}{l}500.3 \\
499.2\end{array}$ & $\begin{array}{l}488.2 \\
486.9\end{array}$ & $\begin{array}{l}716.2 \\
701.1\end{array}$ & $\begin{array}{l}0.357 \\
0.325\end{array}$ & .91 & \\
\hline $\begin{array}{r}58 \\
139\end{array}$ & $\begin{array}{l}98 \\
98\end{array}$ & $\begin{array}{c}H / O \\
H\end{array}$ & $\begin{array}{l}\text { HP } \\
\text { HP }\end{array}$ & $\begin{array}{l}1200 \\
1200\end{array}$ & $\begin{array}{l}2.021 \\
2.019\end{array}$ & $\begin{array}{l}500.7 \\
499.8\end{array}$ & $\begin{array}{l}488.6 \\
487.7\end{array}$ & $\begin{array}{l}665.9 \\
651.3\end{array}$ & $\begin{array}{l}0.556 \\
0.513\end{array}$ & .92 & \\
\hline $\begin{array}{r}60 \\
141\end{array}$ & $\begin{array}{l}98 \\
98\end{array}$ & $W / O$ & $\begin{array}{l}\text { HP } \\
\text { HP }\end{array}$ & $\begin{array}{l}1200 \\
1200\end{array}$ & $\begin{array}{l}2.297 \\
2.823\end{array}$ & $\begin{array}{l}499.6 \\
500.1\end{array}$ & $\begin{array}{l}487.4 \\
488.0\end{array}$ & $\begin{array}{l}652.6 \\
625.7\end{array}$ & $\begin{array}{l}0.589 \\
0.604\end{array}$ & 1.03 & Used on Plot \\
\hline
\end{tabular}


TABLE 2

Comparison of Data With and Without A Hot Patch -2.25 Heat Flux Ratio

\begin{tabular}{|c|c|c|c|c|c|c|c|c|c|}
\hline \multirow[b]{2}{*}{$\begin{array}{l}\text { RUN } \\
\text { NO. }\end{array}$} & & & & & & & & & \\
\hline & TYPE & & $\begin{array}{l}\text { PRES - } \\
\text { (PSIA) }\end{array}$ & $\begin{array}{l}\text { MASS } \\
\text { VELOCITY } \\
\text { X 1B-6 } \\
\text { (LE/HR- } \\
\text { FTSA) }\end{array}$ & $\begin{array}{c}\text { INLET } \\
\text { TEMPER- } \\
\text { ATURE } \\
(\text { DEG.F•) }\end{array}$ & $\begin{array}{l}\text { MEASURED } \\
\text { INLET } \\
\text { ENTHALPY } \\
\text { (BTULLB) }\end{array}$ & $\begin{array}{l}\text { CALCU- } \\
\text { LATED } \\
\text { EXIT } \\
\text { ENTHALPY } \\
\text { (BTU/LB) }\end{array}$ & $\begin{array}{l}\text { AVERAGE } \\
\text { HEAT FLUX } \\
\text { X 10-6 } \\
\text { (BTU/HR- } \\
\text { FTSQ) }\end{array}$ & $\begin{array}{c}\text { RATIO } \\
\text { OF } \\
\text { CRITICAL } \\
\text { HEAT } \\
\text { FLUXES }\end{array}$ \\
\hline $\begin{array}{r}38 \\
209\end{array}$ & $\begin{array}{l}\text { CHF } \quad W / 0 \\
\text { CHF } \quad 2.25\end{array}$ & $\begin{array}{l}H P \\
H P\end{array}$ & $\begin{array}{l}2000 \\
2000\end{array}$ & $\begin{array}{l}0.259 \\
0.254\end{array}$ & $\begin{array}{l}201.5 \\
199.4\end{array}$ & $\begin{array}{l}174.2 \\
172.1\end{array}$ & $\begin{array}{l}779.4 \\
824.9\end{array}$ & $\begin{array}{l}0.244 \\
0.257\end{array}$ & 1.06 \\
\hline $211^{*}$ & $\begin{array}{l}\text { CHF H/O } \\
\text { NO DATA }\end{array}$ & HP & 2000 & 0.499 & $201 \cdot 4$ & 174.1 & 706.8 & 0.413 & \\
\hline $\begin{array}{r}34 \\
217^{*}\end{array}$ & $\begin{array}{l}\text { CHF W/O } \\
\text { NO CATA }\end{array}$ & $H P$ & $200 \mathrm{~J}$ & 0.991 & 200.7 & 173.4 & 635.3 & 0.711 & \\
\hline $\begin{array}{r}27 \\
175\end{array}$ & $\begin{array}{l}\text { CHF } \$ / 0 \\
\text { CHF W } 2.25\end{array}$ & $\begin{array}{l}\text { HP } \\
\text { HP }\end{array}$ & $\begin{array}{l}2000 \\
200 J\end{array}$ & $\begin{array}{l}0.245 \\
0.255\end{array}$ & $\begin{array}{l}401 \cdot 8 \\
397 \cdot 3\end{array}$ & $\begin{array}{l}379.2 \\
374.4\end{array}$ & $\begin{array}{l}858.3 \\
879.8\end{array}$ & $\begin{array}{l}0.182 \\
0.200\end{array}$ & 1.10 \\
\hline $\begin{array}{r}29 \\
173^{*}\end{array}$ & $\begin{array}{l}\text { CHF W/O } \\
\text { NO DATA }\end{array}$ & HP & 2003 & 0.500 & 401.7 & 379.1 & 783.8 & 0.314 & \\
\hline $\begin{array}{r}31 \\
177\end{array}$ & $\begin{array}{l}\text { CHF } \quad H / 0 \\
\text { CHF W } 2.25\end{array}$ & $\begin{array}{l}\text { HP } \\
\text { HP }\end{array}$ & $\begin{array}{l}2000 \\
2000\end{array}$ & $\begin{array}{l}1.000 \\
0.998\end{array}$ & $\begin{array}{l}398.9 \\
400.8\end{array}$ & $\begin{array}{l}376.1 \\
378.2\end{array}$ & $\begin{array}{l}695 \cdot 5 \\
676 \cdot 3\end{array}$ & $\begin{array}{l}0.496 \\
0.462\end{array}$ & .93 \\
\hline $213^{\star \star \star}$ & $\begin{array}{l}\text { CHF W/O } \\
\text { NO DATA }\end{array}$ & HP & 2000 & 1.989 & 400.8 & 378.1 & 609.6 & 0.716 & \\
\hline $\begin{array}{r}25 \\
181\end{array}$ & $\begin{array}{l}\text { CHF } \quad W / 0 \\
\text { CHF } W 2.25\end{array}$ & $\begin{array}{l}\text { HP } \\
\text { HP }\end{array}$ & $\begin{array}{l}2000 \\
2000\end{array}$ & $\begin{array}{l}0.244 \\
0.252\end{array}$ & $\begin{array}{l}501.4 \\
499.8\end{array}$ & $\begin{array}{l}489.3 \\
487.5\end{array}$ & $\begin{array}{l}885.7 \\
892.5\end{array}$ & $\begin{array}{l}0.150 \\
0.159\end{array}$ & 1.06 \\
\hline $\begin{array}{r}23 \\
191\end{array}$ & $\begin{array}{l}\text { CHF } W / C \\
\text { CHF } W 2.25\end{array}$ & $\begin{array}{l}\text { HP } \\
\text { HF }\end{array}$ & $\begin{array}{l}2000 \\
2000\end{array}$ & $\begin{array}{l}0.498 \\
0.501\end{array}$ & $\begin{array}{l}499.7 \\
500.5\end{array}$ & $\begin{array}{l}487.4 \\
488.4\end{array}$ & $\begin{array}{l}800.1 \\
786.7\end{array}$ & $\begin{array}{l}0.242 \\
0.232\end{array}$ & .96 \\
\hline
\end{tabular}

* No data taken at CHF levels, see Runs 212, 218 and 174 at 98 CHF levels.

* Maximum generator (no CHF) was reached. 
TABLE 2 (Continued)

Comparison of Irata With and Without A Hot Patch -2.25 Heat Flux Ratio

\begin{tabular}{|c|c|c|c|c|c|c|c|c|c|}
\hline & & & & & & & & & \\
\hline $\begin{array}{l}\text { RUN } \\
\text { NO. }\end{array}$ & TYPE & & $\begin{array}{l}\text { PRES- } \\
\text { (PSIA) }\end{array}$ & $\begin{array}{l}\text { MASS } \\
\text { VELOCITY } \\
X 10-6 \\
\text { (LB/HR- } \\
\text { FTSQ) }\end{array}$ & $\begin{array}{l}\text { INLET } \\
\text { TEMPER- } \\
\text { ATURE } \\
\text { (OEG.F.) }\end{array}$ & $\begin{array}{l}\text { MEASURED } \\
\text { INLET } \\
\text { ENTHALPY } \\
\text { (BTU/LB) }\end{array}$ & $\begin{array}{l}\text { CALCU- } \\
\text { LATED } \\
\text { EXIT } \\
\text { ENTHALPY } \\
\text { (BTU/LB) }\end{array}$ & $\begin{array}{l}\text { AVERAGE } \\
\text { HEAT FLUX } \\
\text { X 10-6 } \\
\text { (BTU/HR- } \\
\text { FTSQ) }\end{array}$ & $\begin{array}{l}\text { RATIO } \\
\text { OF } \\
\text { CRITICAL } \\
\text { HEAT } \\
\text { FLUXES }\end{array}$ \\
\hline $\begin{array}{r}21 \\
193\end{array}$ & $\begin{array}{l}\text { CHF } \quad W / 0 \\
\text { CHF } W 2.25\end{array}$ & $\begin{array}{l}\text { HP } \\
\text { HP }\end{array}$ & $\begin{array}{l}2000 \\
2000\end{array}$ & $\begin{array}{l}1.000 \\
1.000\end{array}$ & $\begin{array}{l}500.4 \\
500.1\end{array}$ & $\begin{array}{l}488.2 \\
487.9\end{array}$ & $\begin{array}{l}747.3 \\
723.6\end{array}$ & $\begin{array}{l}0.402 \\
0.366\end{array}$ & .91 \\
\hline $223^{19}$ & $\begin{array}{l}\text { CHF H/O } \\
\text { NO DATA }\end{array}$ & HP & 2003 & 1.990 & 500.2 & 488.0 & 690.2 & 0.625 & \\
\hline $\begin{array}{r}18 \\
229\end{array}$ & $\begin{array}{l}\text { CHF } \quad W / 0 \\
\text { CHF } W 2.25\end{array}$ & $\begin{array}{l}H P \\
H P\end{array}$ & $\begin{array}{l}2000 \\
2000\end{array}$ & $\begin{array}{l}2.6 .82 \\
2.810\end{array}$ & $\begin{array}{l}501.1 \\
499.7\end{array}$ & $\begin{array}{l}489.0 \\
487.5\end{array}$ & $\begin{array}{l}660.1 \\
639.9\end{array}$ & $\begin{array}{l}0.713 \\
0.666\end{array}$ & .93 \\
\hline $\begin{array}{r}8 \\
205\end{array}$ & $\begin{array}{l}\text { CHF } W / 0 \\
\text { CHF } W 2.25\end{array}$ & $\begin{array}{l}H P \\
H P\end{array}$ & $\begin{array}{l}2000 \\
2000\end{array}$ & $\begin{array}{l}0.246 \\
0.250\end{array}$ & $\begin{array}{l}596.2 \\
601.4\end{array}$ & $\begin{array}{l}609.3 \\
617.0\end{array}$ & $\begin{array}{l}916.0 \\
963 . E\end{array}$ & $\begin{array}{l}0.117 \\
0.135\end{array}$ & 1.15 \\
\hline $\begin{array}{r}10 \\
207\end{array}$ & $\begin{array}{l}\text { CHF } \quad H / 0 \\
\text { CHF } \quad 2.25\end{array}$ & $\begin{array}{l}\text { HP } \\
\text { HP }\end{array}$ & $\begin{array}{l}2000 \\
2000\end{array}$ & $\begin{array}{l}0.493 \\
0.493\end{array}$ & $\begin{array}{l}599.7 \\
599.7\end{array}$ & $\begin{array}{l}514.6 \\
514.5\end{array}$ & $\begin{array}{l}822.8 \\
828.9\end{array}$ & $\begin{array}{l}0.159 \\
0.164\end{array}$ & 1.03 \\
\hline $\begin{array}{r}12 \\
199\end{array}$ & $\begin{array}{l}\text { CHF } H / C \\
\text { CHF } \quad 2.25\end{array}$ & $\begin{array}{l}\text { HP } \\
\text { HP }\end{array}$ & $\begin{array}{l}2000 \\
2000\end{array}$ & $\begin{array}{l}1.013 \\
1.023\end{array}$ & $\begin{array}{l}599.7 \\
599.7\end{array}$ & $\begin{array}{l}.614 .8 \\
.614 .6\end{array}$ & $\begin{array}{l}772.5 \\
765.1\end{array}$ & $\begin{array}{l}0.248 \\
0.239\end{array}$ & .96 \\
\hline $\begin{array}{r}14 \\
201\end{array}$ & $\begin{array}{l}\text { CHF } \quad H / O \\
\text { CHF } W 2.25\end{array}$ & $\begin{array}{l}\text { HP } \\
\text { HP }\end{array}$ & $\begin{array}{l}2000 \\
2000\end{array}$ & $\begin{array}{l}1.986 \\
2.007\end{array}$ & $\begin{array}{l}599.4 \\
599.7\end{array}$ & $\begin{array}{l}514.1 \\
614.8\end{array}$ & $\begin{array}{l}738.6 \\
731.4\end{array}$ & $\begin{array}{l}0.384 \\
0.364\end{array}$ & .95 \\
\hline $\begin{array}{r}16 \\
203\end{array}$ & $\begin{array}{l}\text { CHF } \quad W / 0 \\
\text { CHF } W 2.25\end{array}$ & $\begin{array}{l}\text { HP } \\
\text { HP }\end{array}$ & $\begin{array}{l}2000 \\
2000\end{array}$ & $\begin{array}{l}2.556 \\
2.690\end{array}$ & $\begin{array}{l}600.2 \\
600.4\end{array}$ & $\begin{array}{l}515.3 \\
515.5\end{array}$ & $\begin{array}{l}723.4 \\
717.3\end{array}$ & $\begin{array}{l}0.429 \\
0.425\end{array}$ & .99 \\
\hline
\end{tabular}

* No data taken at CHP level, see Run 224 at $98 \mathrm{CHF}$ level. 
TABLE 2 (Continued)

Comparison of Data With and Without A Hot Patch - 2.25 Heat Flux Ratio

\begin{tabular}{|c|c|c|c|c|c|c|c|c|c|c|}
\hline $\begin{array}{l}\text { RUN } \\
N C .\end{array}$ & & TYFE & . & $\begin{array}{l}\text { PFES- } \\
\text { (PSIA) }\end{array}$ & $\begin{array}{l}\text { MASS } \\
\text { VELOCITY } \\
\text { X 10-6 } \\
\text { (LB/HR- } \\
\text { FTSQ) }\end{array}$ & $\begin{array}{l}\text { INLET } \\
\text { TEMPER- } \\
\text { ATURE } \\
\text { (DEG.F.) }\end{array}$ & $\begin{array}{l}\text { MEASURED } \\
\text { INLET } \\
\text { ENTHALPY } \\
\text { (BTU/LB) }\end{array}$ & $\begin{array}{l}\text { CALCU- } \\
\text { LATEO } \\
\text { EXIT } \\
\text { ENTHALPY } \\
\text { (BTU/LB) }\end{array}$ & $\begin{array}{l}\text { AVERAGE } \\
\text { HEAT FLUX } \\
\times 10-6 \\
\text { (日TU/HR- } \\
\text { FTSO) }\end{array}$ & $\begin{array}{l}\text { RATIO } \\
\text { OF } \\
\text { CRIT ICAL } \\
\text { HEAT } \\
\text { FLUXES }\end{array}$ \\
\hline $\begin{array}{r}71 \\
167\end{array}$ & $\begin{array}{l}\text { CHF } \\
\text { CHF }\end{array}$ & $\begin{array}{c}W / C \\
W 2.25\end{array}$ & $\begin{array}{l}H P \\
H P\end{array}$ & $\begin{array}{l}1600 \\
1 \in 00\end{array}$ & $\begin{array}{l}0.250 \\
0.249\end{array}$ & $\begin{array}{l}402.5 \\
400.4\end{array}$ & $\begin{array}{l}379.5 \\
377.3\end{array}$ & $\begin{array}{l}842.5 \\
847.6\end{array}$ & $\begin{array}{l}0.180 \\
0.182\end{array}$ & 1.01 \\
\hline $\begin{array}{r}73 \\
169\end{array}$ & $\begin{array}{l}\text { CHF } \\
\text { CHF }\end{array}$ & $\begin{array}{c}W / O \\
W^{\prime} 2.25\end{array}$ & $\begin{array}{l}H P \\
\text { HP }\end{array}$ & $\begin{array}{l}1600 \\
1600\end{array}$ & $\begin{array}{l}0.501 \\
0.499\end{array}$ & $\begin{array}{l}401.3 \\
400.5\end{array}$ & $\begin{array}{l}378.3 \\
377.4\end{array}$ & $\begin{array}{l}728.3 \\
740.7\end{array}$ & $\begin{array}{l}0.272 \\
0.281\end{array}$ & 1.03 \\
\hline $\begin{array}{r}75 \\
171\end{array}$ & $\begin{array}{l}\text { CHF } \\
\text { CHF }\end{array}$ & $\begin{array}{l}W / 0 \\
W 2.25\end{array}$ & $\begin{array}{l}\text { HP } \\
\text { HP }\end{array}$ & $\begin{array}{l}1 \in 0 J \\
1 \in 00\end{array}$ & $\begin{array}{l}1.010 \\
0.998\end{array}$ & $\begin{array}{l}400.6 \\
400.2\end{array}$ & $\begin{array}{l}377.5 \\
377.1\end{array}$ & $\begin{array}{l}681.8 \\
685.5\end{array}$ & $\begin{array}{l}0.478 \\
0.478\end{array}$ & 1.00 \\
\hline $\begin{array}{r}77 \\
221\end{array}$ & $\begin{array}{l}\text { CHF } \\
\text { CHF }\end{array}$ & $\begin{array}{l}H / 0 \\
H \quad 2.25\end{array}$ & $\begin{array}{l}\text { HP } \\
\text { HP }\end{array}$ & $\begin{array}{l}1600 \\
1600\end{array}$ & $\begin{array}{l}2.003 \\
1.987\end{array}$ & $\begin{array}{l}401.0 \\
400.7\end{array}$ & $\begin{array}{l}378.0 \\
377.6\end{array}$ & $\begin{array}{l}610 \cdot 4 \\
606 \cdot 3\end{array}$ & $\begin{array}{l}0.723 \\
0.706\end{array}$ & .98 \\
\hline $\begin{array}{r}69 \\
183\end{array}$ & $\begin{array}{l}\text { CHF } \\
\text { CHF }\end{array}$ & $\begin{array}{l}W / O \\
W 2.25\end{array}$ & $\begin{array}{l}\text { HP } \\
\text { HP }\end{array}$ & $\begin{array}{l}1603 \\
1600\end{array}$ & $\begin{array}{l}0.250 \\
0.251\end{array}$ & $\begin{array}{l}499.3 \\
500.7\end{array}$ & $\begin{array}{l}486.9 \\
488.5\end{array}$ & $\begin{array}{l}868.4 \\
881.8\end{array}$ & $\begin{array}{l}0.148 \\
0.153\end{array}$ & 1.03 \\
\hline $\begin{array}{r}67 \\
189\end{array}$ & $\begin{array}{l}\text { CHF } \\
\text { CHF }\end{array}$ & $\begin{array}{c}W / C \\
W \quad 2.25\end{array}$ & $\begin{array}{l}H P \\
H P\end{array}$ & $\begin{array}{l}1 \in 00 \\
1 \in 00\end{array}$ & $\begin{array}{l}0.508 \\
0.502\end{array}$ & $\begin{array}{l}499.7 \\
500.8\end{array}$ & $\begin{array}{l}487.4 \\
488.6\end{array}$ & $\begin{array}{l}763.4 \\
781.5\end{array}$ & $\begin{array}{l}0.218 \\
0.228\end{array}$ & 1.05 \\
\hline $\begin{array}{r}65 \\
195\end{array}$ & $\begin{array}{l}\text { CHF } \\
\text { CHF }\end{array}$ & $\begin{array}{l}W / C \\
W \quad 2.25\end{array}$ & $\begin{array}{l}\text { HP } \\
\text { HP }\end{array}$ & $\begin{array}{l}1 E 00 \\
1603\end{array}$ & $\begin{array}{l}0.997 \\
0.974\end{array}$ & $\begin{array}{l}499.7 \\
500.1\end{array}$ & $\begin{array}{l}487.3 \\
487.8\end{array}$ & $\begin{array}{l}708.2 \\
709.3\end{array}$ & $\begin{array}{l}0.342 \\
0.335\end{array}$ & .98 \\
\hline $\begin{array}{r}63 \\
225\end{array}$ & $\begin{array}{l}\text { CHF } \\
\text { CHF }\end{array}$ & $\begin{array}{l}W / 0 \\
W \quad 2.25\end{array}$ & $\begin{array}{l}\text { HP } \\
\text { HP }\end{array}$ & $\begin{array}{l}1 \in 00 \\
1 \in 00\end{array}$ & $\begin{array}{l}1.989 \\
1.969\end{array}$ & $\begin{array}{l}500 \cdot 1 \\
500 \cdot 0\end{array}$ & $\begin{array}{l}487.8 \\
487.6\end{array}$ & $\begin{array}{l}675.6 \\
667.4\end{array}$ & $\begin{array}{l}0.580 \\
0.550\end{array}$ & .95 \\
\hline $\begin{array}{r}61^{*} \\
227\end{array}$ & $\begin{array}{l}\text { NO } \\
\text { CHF }\end{array}$ & $\begin{array}{l}\text { DATA } \\
\text { W } 2.25\end{array}$ & HP & 1600 & 2.772 & 500.5 & 488.2 & $632 \cdot 1$ & 0.620 & \\
\hline
\end{tabular}

* No data taken at CHF level, see Run 62 at $98 \mathrm{CHF}$ level. 
TABLE 2 (Continued)

Comparison of Data With and Without A Hot Patch -2.25 Heat Flux Ratio

\begin{tabular}{|c|c|c|c|c|c|c|c|c|c|c|c|}
\hline $\begin{array}{l}\text { RUN } \\
\text { NO. }\end{array}$ & & TYPE & & $\begin{array}{l}\text { PRES- } \\
\text { (PSIA) }\end{array}$ & $\begin{array}{l}\text { MASS } \\
\text { VELOCITY } \\
\times 10-E \\
\text { (LB/HR- } \\
\text { FTSQ) }\end{array}$ & $\begin{array}{c}\text { INLET } \\
\text { TEMPER- } \\
\text { ATURE } \\
\text { (DEG.F.) }\end{array}$ & $\begin{array}{l}\text { MEASURED } \\
\text { INLET } \\
\text { ENTHALPY } \\
\text { (BTUルB) }\end{array}$ & $\begin{array}{l}\text { CALCU- } \\
\text { LATEO } \\
\text { EXIT } \\
\text { ENTHALPY } \\
\text { (BTU/LB) }\end{array}$ & $\begin{array}{l}\text { AVERAGE } \\
\text { HEAT FLUX } \\
\text { X 10-6 } \\
\text { (BTU/HR- } \\
\text { FTSQ) }\end{array}$ & $\begin{array}{l}\text { RATIO } \\
\text { OF } \\
\text { CRITICAL } \\
\text { HEAT } \\
\text { FLUXES }\end{array}$ & \\
\hline $\begin{array}{r}40 \\
215\end{array}$ & $\begin{array}{l}\text { CHF } \\
\text { CHF }\end{array}$ & $\begin{array}{l}1 / 0 \\
w 2.25\end{array}$ & $\begin{array}{l}\text { HP } \\
\text { HP }\end{array}$ & $\begin{array}{l}1230 \\
1230\end{array}$ & $\begin{array}{l}0.245 \\
0.255\end{array}$ & $\begin{array}{l}200 \cdot 2 \\
200 \cdot 8\end{array}$ & $\begin{array}{l}171.1 \\
171.6\end{array}$ & $\begin{array}{l}704 \cdot 3 \\
765.9\end{array}$ & $\begin{array}{l}0.203 \\
0.236\end{array}$ & 1.16 & \\
\hline $\begin{array}{r}42 \\
213\end{array}$ & $\begin{array}{l}\text { CHF } \\
\text { CHF }\end{array}$ & $\begin{array}{c}W / 0 \\
W \quad 2.25\end{array}$ & $\begin{array}{l}\text { HP } \\
\text { HP }\end{array}$ & $\begin{array}{l}12.30 \\
1230\end{array}$ & $\begin{array}{l}0.501 \\
0.500\end{array}$ & $\begin{array}{l}199.8 \\
201.1\end{array}$ & $\begin{array}{l}170.6 \\
171.9\end{array}$ & $\begin{array}{l}689.0 \\
650.1\end{array}$ & $\begin{array}{l}0.404 \\
0.372\end{array}$ & .92 & \\
\hline $\begin{array}{r}44 \\
160\end{array}$ & $\begin{array}{l}\text { CHF } \\
\text { CHF }\end{array}$ & $\begin{array}{l}W / 0 \\
\quad 2.25\end{array}$ & $\begin{array}{l}\text { HP } \\
\text { HP }\end{array}$ & $\begin{array}{l}1230 \\
1200\end{array}$ & $\begin{array}{l}0.247 \\
0.247\end{array}$ & $\begin{array}{l}401.0 \\
400.2\end{array}$ & $\begin{array}{l}377.5 \\
376.7\end{array}$ & $\begin{array}{l}814 \cdot 3 \\
845.9\end{array}$ & $\begin{array}{l}0.167 \\
0.18 \mathrm{i}\end{array}$ & 1.08 & \\
\hline $\begin{array}{r}46 \\
1 \in 2\end{array}$ & $\begin{array}{l}\text { CHF } \\
\text { CHF }\end{array}$ & $\begin{array}{l}N / 0 \\
W \quad 2.25\end{array}$ & $\begin{array}{l}\text { HP } \\
H P\end{array}$ & $\begin{array}{l}1200 \\
1203\end{array}$ & $\begin{array}{l}0.493 \\
0.497\end{array}$ & $\begin{array}{l}399.8 \\
400.1\end{array}$ & $\begin{array}{l}376.2 \\
376.6\end{array}$ & $\begin{array}{l}735.5 \\
730.9\end{array}$ & $\begin{array}{l}0.275 \\
0.274\end{array}$ & .99 & \\
\hline $\begin{array}{r}48 \\
1 \in 4\end{array}$ & $\begin{array}{l}\text { CHF } \\
\text { CHF }\end{array}$ & $\begin{array}{l}W / O \\
H \quad 2.25\end{array}$ & $\begin{array}{l}1 A P \\
-1 P\end{array}$ & $\begin{array}{l}1200 \\
1200\end{array}$ & $\begin{array}{l}0.921 \\
0.989\end{array}$ & $\begin{array}{l}400.5 \\
400.5\end{array}$ & $\begin{array}{l}377.0 \\
377.0\end{array}$ & $\begin{array}{l}681.4 \\
653.2\end{array}$ & $\begin{array}{l}0.435 \\
0.440\end{array}$ & 1.01 & \\
\hline $\begin{array}{r}48 A \\
164\end{array}$ & $\begin{array}{l}\text { CHF } \\
\text { CHF }\end{array}$ & $\begin{array}{l}W / O \\
W 2.25\end{array}$ & $\begin{array}{l}-P \\
H P\end{array}$ & $\begin{array}{l}1200 \\
1200\end{array}$ & $\begin{array}{l}0.987 \\
0.989\end{array}$ & $\begin{array}{l}400.7 \\
400.5\end{array}$ & $\begin{array}{l}377.2 \\
377.0\end{array}$ & $\begin{array}{l}684 \cdot 6 \\
663 \cdot 2\end{array}$ & $\begin{array}{l}0.471 \\
0.440\end{array}$ & .93 & Repeat \\
\hline $\begin{array}{r}50 \\
166\end{array}$ & $\begin{array}{l}\text { CHF } \\
\text { CHF }\end{array}$ & $\begin{array}{l}w / 0 \\
* 2.25\end{array}$ & $\begin{array}{l}H P \\
-1 P\end{array}$ & $\begin{array}{l}1200 \\
1200\end{array}$ & $\begin{array}{l}2.010 \\
1.991\end{array}$ & $\begin{array}{l}400.9 \\
400.6\end{array}$ & $\begin{array}{l}377.4 \\
377.1\end{array}$ & $\begin{array}{l}509.6 \\
565.4\end{array}$ & $\begin{array}{l}0.725 \\
0.582\end{array}$ & .80 & \\
\hline $\begin{array}{r}51 \\
185\end{array}$ & $\begin{array}{l}\text { CHF } \\
\text { CHF }\end{array}$ & $\begin{array}{l}H / 0 \\
-2.25\end{array}$ & $\begin{array}{l}H P \\
-1 P\end{array}$ & $\begin{array}{l}1200 \\
1200\end{array}$ & $\begin{array}{l}0.248 \\
0.251\end{array}$ & $\begin{array}{l}501.0 \\
497.3\end{array}$ & $\begin{array}{l}489.0 \\
484.8\end{array}$ & $\begin{array}{l}837.4 \\
863.6\end{array}$ & $\begin{array}{l}0.134 \\
0.148\end{array}$ & $1 \cdot 10$ & \\
\hline $\begin{array}{r}53 \\
187\end{array}$ & $\begin{array}{l}\text { CHF } \\
\text { CHF }\end{array}$ & $\begin{array}{l}H / 0 \\
W \quad 2.25\end{array}$ & $\begin{array}{l}H P \\
H P\end{array}$ & $\begin{array}{l}1200 \\
1200\end{array}$ & $\begin{array}{l}0.471 \\
0.499\end{array}$ & $\begin{array}{l}506.7 \\
498.5\end{array}$ & $\begin{array}{l}488.6 \\
486.1\end{array}$ & $\begin{array}{l}756.2 \\
763.5\end{array}$ & $\begin{array}{l}0.196 \\
0.215\end{array}$ & 1.10 & . \\
\hline $\begin{array}{r}55 \\
197\end{array}$ & $\begin{array}{l}\text { CHF } \\
\text { CHF }\end{array}$ & $\begin{array}{l}H / 0 \\
+2.25\end{array}$ & $\begin{array}{l}\text { HP } \\
\text { HP }\end{array}$ & $\begin{array}{l}1200 \\
1200\end{array}$ & $\begin{array}{l}1.003 \\
1.011\end{array}$ & $\begin{array}{l}500 \cdot 2 \\
499.3\end{array}$ & $\begin{array}{l}488.0 \\
487.1\end{array}$ & $\begin{array}{l}723.0 \\
693.4\end{array}$ & $\begin{array}{l}0.366 \\
0.324\end{array}$ & .88 & \\
\hline 57 & CHF & $H / O$ & HP & 1200 & $2.014^{\circ}$ & $500 \cdot 2$ & 488.1 & 667.6 & 0.562 & & \\
\hline
\end{tabular}

No 2.25 hot patch data taken due to generator limitations.

59* NO DATA

No 2.25 hot patch data taken tue to generator limitations

* No data také at chr level, see Table 6 (Run 60) at $98 \mathrm{cHF}$ level. 
TABLE 2 (Continued)

Comparison of Data With and Without $A$ Hot Patch -2.25 Heat Flux Ratio

\begin{tabular}{|c|c|c|c|c|c|c|c|c|c|c|c|c|c|}
\hline $\begin{array}{l}\text { RUN } \\
\text { NO. }\end{array}$ & & TYPE & & $\begin{array}{l}\text { PRES- } \\
\text { (PSIA) }\end{array}$ & $\begin{array}{l}\text { MASS } \\
\text { VELOCITY } \\
\text { X 10-6 } \\
\text { (LB/HR- } \\
\text { FTSO) }\end{array}$ & $\begin{array}{l}\text { INLET } \\
\text { TEMPER- } \\
\text { ATURE } \\
\text { (OEG.F•) }\end{array}$ & $\begin{array}{l}\text { MEASURED } \\
\text { INLET } \\
\text { ENTHALPY } \\
\text { (BTLAB) }\end{array}$ & $\begin{array}{l}\text { CALCU- } \\
\text { LATED } \\
\text { EXIT } \\
\text { ENTHALPY } \\
\text { (BTUILB) }\end{array}$ & $\begin{array}{l}\text { AVERAGE } \\
\text { HEAT FLUX } \\
\text { X 10-6 } \\
\text { (BTU/HR- } \\
\text { FTSO) }\end{array}$ & $\begin{array}{l}\text { RATIO } \\
\text { OF } \\
\text { CRITICAL } \\
\text { HEAT } \\
\text { FLUXES }\end{array}$ & & & \\
\hline $\begin{array}{r}39 \\
210\end{array}$ & $\begin{array}{l}98 \\
98\end{array}$ & $\begin{array}{l}4 / 0 \\
H 2.25\end{array}$ & $\begin{array}{l}\text { HP } \\
\text { HP }\end{array}$ & $\begin{array}{l}2000 \\
2000\end{array}$ & $\begin{array}{l}6.258 \\
0.254\end{array}$ & $\begin{array}{l}201.6 \\
199.4\end{array}$ & $\begin{array}{l}174 \cdot 3 \\
172 \cdot 0\end{array}$ & $\begin{array}{l}769 \cdot 8 \\
809 \cdot 2\end{array}$ & $\begin{array}{l}0.239 \\
0.252\end{array}$ & 1.05 & & & \\
\hline $\begin{array}{r}37 \\
212\end{array}$ & $\begin{array}{l}98 \\
98\end{array}$ & $\begin{array}{l}H / 0 \\
W 2.25\end{array}$ & $\begin{array}{l}\text { HP } \\
\text { HP }\end{array}$ & $\begin{array}{l}2000 \\
2000\end{array}$ & $\begin{array}{l}0.498 \\
0.497\end{array}$ & $\begin{array}{l}201 \cdot 6 \\
201 \cdot 1\end{array}$ & $\begin{array}{l}174.3 \\
173.8\end{array}$ & $\begin{array}{l}695 \cdot 8 \\
711.1\end{array}$ & $\begin{array}{l}0.403 \\
0.415\end{array}$ & 1.03 & Used & in & Plot \\
\hline $\begin{array}{r}35 \\
218\end{array}$ & $\begin{array}{l}98 \\
98\end{array}$ & $\begin{array}{l}H / 0 \\
H \quad 2.25\end{array}$ & $\begin{array}{l}\text { HP } \\
\text { HP }\end{array}$ & $\begin{array}{l}2000 \\
2000\end{array}$ & $\begin{array}{l}0.991 \\
1.002\end{array}$ & $\begin{array}{l}200.7 \\
200.6\end{array}$ & $\begin{array}{l}173.4 \\
173.2\end{array}$ & $\begin{array}{l}625.2 \\
559.9\end{array}$ & $\begin{array}{l}0.695 \\
0.601\end{array}$ & .86 & & & \\
\hline $\begin{array}{r}28 \\
176\end{array}$ & $\begin{array}{l}98 \\
98\end{array}$ & $\begin{array}{l}H / 0 \\
W 2.25\end{array}$ & $\begin{array}{l}\text { HP } \\
\text { HP }\end{array}$ & $\begin{array}{l}2000 \\
2000\end{array}$ & $\begin{array}{l}0.244 \\
0.254\end{array}$ & $\begin{array}{l}401.5 \\
399.4\end{array}$ & $\begin{array}{l}378.9 \\
376.6\end{array}$ & $\begin{array}{l}848.5 \\
860.8\end{array}$ & $\begin{array}{l}0.178 \\
0.191\end{array}$ & 1.08 & & & . \\
\hline $\begin{array}{r}30 \\
174\end{array}$ & $\begin{array}{l}98 \\
98\end{array}$ & $\begin{array}{l}4 / 0 \\
W 2.25\end{array}$ & $\begin{array}{l}\text { HP } \\
\text { HP }\end{array}$ & $\begin{array}{l}2000 \\
2000\end{array}$ & $\begin{array}{l}0.500 \\
0.496\end{array}$ & $\begin{array}{l}402.2 \\
400.3\end{array}$ & $\begin{array}{l}379.7 \\
377.6\end{array}$ & $\begin{array}{l}780 \cdot 2 \\
783 \cdot 2\end{array}$ & $\begin{array}{l}8.311 \\
0.313\end{array}$ & 1.00 & Used & in & Plot \\
\hline $\begin{array}{r}32 \\
178\end{array}$ & $\begin{array}{l}98 \\
98\end{array}$ & $\begin{array}{c}W / 0 \\
N 2.25\end{array}$ & $\begin{array}{l}\text { HP } \\
\text { HP }\end{array}$ & $\begin{array}{l}2000 \\
2000\end{array}$ & $\begin{array}{l}1.000 \\
0.998\end{array}$ & $\begin{array}{l}399.0 \\
401.2\end{array}$ & $\begin{array}{l}376 \cdot 2 \\
378 \cdot 6\end{array}$ & $\begin{array}{l}691.8 \\
673.4\end{array}$ & $\begin{array}{l}0.490 \\
0.457\end{array}$ & .93 & & - & \\
\hline $\begin{array}{r}26 \\
182\end{array}$ & $\begin{array}{l}98 \\
98\end{array}$ & $\begin{array}{l}N / C \\
H 2.25\end{array}$ & $\begin{array}{l}\text { HP } \\
\text { HP }\end{array}$ & $\begin{array}{l}2000 \\
2000\end{array}$ & $\begin{array}{l}0.243 \\
0.255\end{array}$ & $\begin{array}{l}502.0 \\
499.8\end{array}$ & $\begin{array}{l}490.1 \\
487.5\end{array}$ & $\begin{array}{l}877 \cdot 1 \\
874.2\end{array}$ & $\begin{array}{l}0.146 \\
0.153\end{array}$ & 1.05 & & & \\
\hline $\begin{array}{r}24 \\
192\end{array}$ & $\begin{array}{l}98 \\
98\end{array}$ & $\begin{array}{l}H / 0 \\
W \quad 2.25\end{array}$ & $\begin{array}{l}H P \\
H P\end{array}$ & $\begin{array}{l}2000 \\
2000\end{array}$ & $\begin{array}{l}0.495 \\
0.502\end{array}$ & $\begin{array}{l}499.6 \\
500.5\end{array}$ & $\begin{array}{l}487.3 \\
488.3\end{array}$ & $\begin{array}{l}794 \cdot 8 \\
776.9\end{array}$ & $\begin{array}{l}0.237 \\
0.225\end{array}$ & .95 & & & \\
\hline
\end{tabular}


TA3LE 2 (Continued)

Ccmparison of Data With and Without A Hot Patch -2.25 Heat Flux Ratio

\begin{tabular}{|c|c|c|c|c|c|c|c|c|c|c|}
\hline $\begin{array}{l}\text { RUN } \\
\text { NO. }\end{array}$ & & TYPE & & $\begin{array}{l}\text { PRES- } \\
\text { (FSIA) }\end{array}$ & $\begin{array}{l}\text { MASS } \\
\text { VELOCITY } \\
\text { X } 10-6 \\
\text { (LB/HR- } \\
\text { FTSQ) }\end{array}$ & $\begin{array}{l}\text { INLET } \\
\text { TEMPER- } \\
\text { ATUKE } \\
\text { (OEG.F.) }\end{array}$ & $\begin{array}{l}\text { MEASURED } \\
\text { INLET } \\
\text { ENTHALPY } \\
\text { (GTU/LE) }\end{array}$ & $\begin{array}{l}\text { CALCU- } \\
\text { LATED } \\
\text { EXIT } \\
\text { ENTHALPY } \\
\text { (BTU/LB) }\end{array}$ & $\begin{array}{l}\text { DVERAGE } \\
\text {-EAT FLUX } \\
\text { X 10-6 } \\
\text { (BTU/HR- } \\
\text { FTSQ) }\end{array}$ & $\begin{array}{c}\text { RATIO } \\
\text { OF } \\
\text { CRITICAL } \\
\text { HEAT } \\
\text { FLUXES }\end{array}$ \\
\hline $\begin{array}{r}22 \\
194\end{array}$ & $\begin{array}{l}98 \\
98\end{array}$ & $\begin{array}{l}w / 0 \\
W \quad 2.25\end{array}$ & $\begin{array}{l}\text { HP } \\
\text { HP }\end{array}$ & $\begin{array}{l}2000 \\
2000\end{array}$ & $\begin{array}{l}1.000 \\
1.000\end{array}$ & $\begin{array}{l}500 \cdot 6 \\
500 \cdot 2\end{array}$ & $\begin{array}{l}488.5 \\
487.9\end{array}$ & $\begin{array}{l}742.8 \\
717.2 .\end{array}$ & $\begin{array}{l}3.395 \\
0.356\end{array}$ & .90 \\
\hline $\begin{array}{r}23 \\
224\end{array}$ & $\begin{array}{l}98 \\
98\end{array}$ & $\begin{array}{l}W / C \\
W 2.25\end{array}$ & $\begin{array}{l}\text { HP } \\
\text { HP }\end{array}$ & $\begin{array}{l}2000 \\
2003\end{array}$ & $\begin{array}{l}1.997 \\
1.995\end{array}$ & $\begin{array}{l}499.6 \\
501.4\end{array}$ & $\begin{array}{l}487.4 \\
489.3\end{array}$ & $\begin{array}{l}634.8 \\
663.4\end{array}$ & $\begin{array}{l}.0612 \\
0.540\end{array}$ & .88 \\
\hline $\begin{array}{r}9 \\
206\end{array}$ & $\begin{array}{l}98 \\
98\end{array}$ & $\begin{array}{l}W / 0 \\
w 2.25\end{array}$ & $\begin{array}{l}H P \\
H P\end{array}$ & $\begin{array}{l}2003 \\
2000\end{array}$ & $\begin{array}{l}0.248 \\
0.251\end{array}$ & $\begin{array}{l}596.1 \\
500.9\end{array}$ & $\begin{array}{l}609.3 \\
616.3\end{array}$ & $\begin{array}{l}904 \cdot 9 \\
953.5\end{array}$ & $\begin{array}{l}3.114 \\
0.132\end{array}$ & 1.15 \\
\hline $\begin{array}{r}11 \\
208\end{array}$ & $\begin{array}{l}98 \\
98\end{array}$ & $\begin{array}{l}w / 0 \\
w 2.25\end{array}$ & $\begin{array}{l}H P \\
H P\end{array}$ & $\begin{array}{l}2000 \\
2000\end{array}$ & $\begin{array}{l}0.492 \\
0.492\end{array}$ & $\begin{array}{l}599.6 \\
599.7\end{array}$ & $\begin{array}{l}614.4 \\
614.5\end{array}$ & $\begin{array}{l}818.9 \\
824.3\end{array}$ & $\begin{array}{l}0.156 \\
0.16 C\end{array}$ & 1.03 \\
\hline $\begin{array}{r}13 \\
200\end{array}$ & $\begin{array}{l}98 \\
98\end{array}$ & $\begin{array}{l}W / 0 \\
W 2.25\end{array}$ & $\begin{array}{l}H P \\
H P\end{array}$ & $\begin{array}{l}2000 \\
2000\end{array}$ & $\begin{array}{l}1.013 \\
1.019\end{array}$ & $\begin{array}{l}599.5 \\
599.6\end{array}$ & $\begin{array}{l}614.2 \\
614.3\end{array}$ & $\begin{array}{l}768.6 \\
760.0\end{array}$ & $\begin{array}{l}D .243 \\
0.231\end{array}$ & .95 \\
\hline $\begin{array}{r}15 \\
202\end{array}$ & $\begin{array}{l}98 \\
98\end{array}$ & $\begin{array}{l}W / 0 \\
W 2.25\end{array}$ & $\begin{array}{l}H P \\
H P\end{array}$ & $\begin{array}{l}2000 \\
2000\end{array}$ & $\begin{array}{l}1.983 \\
2.011\end{array}$ & $\begin{array}{l}599.0 \\
599.5\end{array}$ & $\begin{array}{l}613.5 \\
614.2\end{array}$ & $\begin{array}{l}736.2 \\
726.7\end{array}$ & $\begin{array}{l}\text { D. } 378 \\
\text { D. } 351\end{array}$ & .93 \\
\hline $\begin{array}{r}17 \\
204\end{array}$ & $\begin{array}{l}98 \\
98\end{array}$ & $\begin{array}{c}W / C \\
W 2.25\end{array}$ & $\begin{array}{l}\text { HF } \\
\text { HP }\end{array}$ & $\begin{array}{l}2000 \\
2000\end{array}$ & $\begin{array}{l}2.561 \\
2.694\end{array}$ & $\begin{array}{l}599.5 \\
600.0\end{array}$ & $\begin{array}{l}614.3 \\
615.0\end{array}$ & $\begin{array}{l}720.1 \\
713.2\end{array}$ & $\begin{array}{l}0.421 \\
0.411\end{array}$ & .98 \\
\hline
\end{tabular}


TABLE 2 (Continued)

Comparison of Data With and Without A Hot Patch - 2.25 Heat Flux Ratio

\begin{tabular}{|c|c|c|c|c|c|c|c|c|c|c|}
\hline $\begin{array}{l}\text { RUN } \\
\text { NO. }\end{array}$ & & TYPE & & $\begin{array}{l}\text { PRES- } \\
\text { (PSIA) }\end{array}$ & $\begin{array}{l}X 1 Q-6 \\
(L B / H R- \\
\text { FTSQ) }\end{array}$ & $\begin{array}{c}\text { TEMPER- } \\
\text { ATURE } \\
\text { (OEG.F.) }\end{array}$ & $\begin{array}{l}\text { INLET } \\
\text { ENTHALPY } \\
\text { (BTU/LB) }\end{array}$ & $\begin{array}{l}\text { EXIT } \\
\text { ENTHALPY } \\
\text { (BTU/LB) }\end{array}$ & $\begin{array}{l}X 10-6 \\
\text { (BTU/HR- } \\
\text { FTSQ) }\end{array}$ & $\begin{array}{c}\text { CRIT ICAL } \\
\text { HE AT } \\
\text { FLUXES }\end{array}$ \\
\hline $\begin{array}{r}72 \\
168\end{array}$ & $\begin{array}{l}98 \\
98\end{array}$ & $\begin{array}{l}W / 0 \\
W \quad 2.25\end{array}$ & $\begin{array}{l}\text { HP } \\
\text { HP }\end{array}$ & $\begin{array}{l}1603 \\
1600\end{array}$ & $\begin{array}{l}0.250 \\
0.248\end{array}$ & $\begin{array}{l}402.5 \\
400.4\end{array}$ & $\begin{array}{l}379.5 \\
377.3\end{array}$ & $\begin{array}{l}821.6 \\
828.2\end{array}$ & $\begin{array}{l}0.172 \\
0.174\end{array}$ & 1.01 \\
\hline $\begin{array}{r}74 \\
170\end{array}$ & $\begin{array}{l}98 \\
38\end{array}$ & $\begin{array}{c}W / 0 \\
W 2.25\end{array}$ & $\begin{array}{l}\text { HP } \\
\text { HP }\end{array}$ & $\begin{array}{l}1600 \\
1 \in 00\end{array}$ & $\begin{array}{l}0.502 \\
0.499\end{array}$ & $\begin{array}{l}401.1 \\
400.5\end{array}$ & $\begin{array}{l}378.0 \\
377.4\end{array}$ & $\begin{array}{l}722 \cdot 4 \\
727.3\end{array}$ & $\begin{array}{l}0.269 \\
0.271\end{array}$ & 1.01 \\
\hline $\begin{array}{r}76 \\
172\end{array}$ & $\begin{array}{l}98 \\
98\end{array}$ & $\begin{array}{l}N / 0 \\
W 2.25\end{array}$ & $\begin{array}{l}\text { HP } \\
\text { HP }\end{array}$ & $\begin{array}{l}1 \in 00 \\
1 \in 00\end{array}$ & $\begin{array}{l}1.013 \\
1.000\end{array}$ & $\begin{array}{l}400.8 \\
400.4\end{array}$ & $\begin{array}{l}377.7 \\
377.2\end{array}$ & $\begin{array}{l}677.4 \\
666.2\end{array}$ & $\begin{array}{l}0.471 \\
0.449\end{array}$ & .95 \\
\hline $\begin{array}{r}78 \\
222\end{array}$ & $\begin{array}{l}\text { CHF } \\
98\end{array}$ & $\begin{array}{l}H / O \\
W 2.25\end{array}$ & $\begin{array}{l}H P \\
H P\end{array}$ & $\begin{array}{l}1600 \\
1600\end{array}$ & $\begin{array}{l}1.985 \\
1.985\end{array}$ & $\begin{array}{l}400.1 \\
400.2\end{array}$ & $\begin{array}{l}376.6 \\
377.1\end{array}$ & $\begin{array}{l}612.9 \\
599.4\end{array}$ & $\begin{array}{l}0.728 \\
0.686\end{array}$ & .94 \\
\hline $\begin{array}{r}70 \\
184\end{array}$ & $\begin{array}{l}98 \\
98\end{array}$ & $\begin{array}{l}W / 0 \\
W \quad 2.25\end{array}$ & $\begin{array}{l}\text { HP } \\
\text { HP }\end{array}$ & $\begin{array}{l}1600 \\
1600\end{array}$ & $\begin{array}{l}0.251 \\
0.250\end{array}$ & $\begin{array}{l}499.4 \\
499.4\end{array}$ & $\begin{array}{l}487.0 \\
486.9\end{array}$ & $\begin{array}{l}854.5 \\
863.3\end{array}$ & $\begin{array}{l}0.143 \\
0.146\end{array}$ & 1.02 \\
\hline $\begin{array}{r}68 \\
190\end{array}$ & $\begin{array}{l}98 \\
98\end{array}$ & $\begin{array}{l}w / 0 \\
w 2.25\end{array}$ & $\begin{array}{l}\text { HP } \\
\text { HP }\end{array}$ & $\begin{array}{l}1600 \\
1600\end{array}$ & $\begin{array}{l}0.507 \\
0.502\end{array}$ & $\begin{array}{l}499.5 \\
500.5\end{array}$ & $\begin{array}{l}487.1 \\
488.2\end{array}$ & $\begin{array}{l}756.0 \\
772.7\end{array}$ & $\begin{array}{l}0.212 \\
0.222\end{array}$ & 1.05 \\
\hline $\begin{array}{r}66 \\
196\end{array}$ & $\begin{array}{l}98 \\
98\end{array}$ & $\begin{array}{l}w / 0 \\
w 2.25\end{array}$ & $\begin{array}{l}H P \\
H P\end{array}$ & $\begin{array}{l}1 \in 00 \\
1 \in 00 .\end{array}$ & $\begin{array}{l}0.996 \\
0.973\end{array}$ & $\begin{array}{l}500.0 \\
499.5\end{array}$ & $\begin{array}{l}487.6 \\
487.1\end{array}$ & $\begin{array}{l}704.8 \\
703.1\end{array}$ & $\begin{array}{l}0.336 \\
0.327\end{array}$ & .97 \\
\hline $\begin{array}{r}54 \\
22 E\end{array}$ & $\begin{array}{l}98 \\
98\end{array}$ & $\begin{array}{l}W / 0 \\
W 2.25\end{array}$ & $\begin{array}{l}\text { HP } \\
\text { HP }\end{array}$ & $\begin{array}{l}1600 \\
1600\end{array}$ & $\begin{array}{l}1.998 \\
1.976\end{array}$ & $\begin{array}{l}500.4 \\
500.5\end{array}$ & $\begin{array}{l}488.1 \\
488.3\end{array}$ & $\begin{array}{l}671.7 \\
662.1\end{array}$ & $\begin{array}{l}0.570 \\
0.533\end{array}$ & .94 \\
\hline $\begin{array}{r}52 \\
228\end{array}$ & $\begin{array}{l}98 \\
98\end{array}$ & $\begin{array}{c}W / 0 \\
W 2.25\end{array}$ & $\begin{array}{l}\text { HP } \\
\text { HP }\end{array}$ & $\begin{array}{l}1 \in O 0 \\
1 \in 00\end{array}$ & $\begin{array}{l}2.56 .2 \\
2.780\end{array}$ & $\begin{array}{l}499.7 \\
500.5\end{array}$ & $\begin{array}{l}487.4 \\
488.3\end{array}$ & $\begin{array}{l}660.6 \\
635.2\end{array}$ & $\begin{array}{l}0.690 \\
0.634\end{array}$ & .92 \\
\hline
\end{tabular}


TABLE 2 (Continued)

Comparison of Data With and W-thout A Hot Patch - 2.25 Heat Flux Ratio

\begin{tabular}{|c|c|c|c|c|c|c|c|c|c|c|}
\hline $\begin{array}{l}\text { RUN } \\
\text { NO. }\end{array}$ & & TYPE & & $\begin{array}{l}\text { PRES- } \\
\text { (PSIA) }\end{array}$ & $\begin{array}{l}\text { MASS } \\
\text { VELOCITY } \\
X 10-6 \\
\text { (LB/HR- } \\
\text { FTSQ) }\end{array}$ & $\begin{array}{c}\text { INLET } \\
\text { TEMPER- } \\
\text { ATURE } \\
\text { (DEG,F.) }\end{array}$ & $\begin{array}{l}\text { MEASURED } \\
\text { INLET } \\
\text { ENTHALPY } \\
\text { (BTU/LB) }\end{array}$ & $\begin{array}{l}\text { CALCU- } \\
\text { LATED } \\
\text { EXIT } \\
\text { ENTHALPY } \\
\text { (BTU/LB) }\end{array}$ & $\begin{array}{l}\text { AVERAGE } \\
\text { HEAT FLUX } \\
\text { X 10-6 } \\
\text { (BTU/HR- } \\
\text { FTSQ) }\end{array}$ & $\begin{array}{l}\text { RAJIO } \\
\text { OF } \\
\text { CRITICAL } \\
\text { HEAT } \\
\text { FLUXES }\end{array}$ \\
\hline $\begin{array}{r}41 \\
216\end{array}$ & $\begin{array}{l}98 \\
98\end{array}$ & $\begin{array}{l}H / 0 \\
H \quad 2.25\end{array}$ & $\begin{array}{l}H P \\
H P\end{array}$ & $\begin{array}{l}1200 \\
1200\end{array}$ & $\begin{array}{l}0 . \geq 45 \\
0 . \geq 55\end{array}$ & $\begin{array}{l}200.2 \\
200.7\end{array}$ & $\begin{array}{l}171.0 \\
171.5\end{array}$ & $\begin{array}{l}677.3 \\
746.2\end{array}$ & $\begin{array}{l}0.193 \\
0.228\end{array}$ & $1 \cdot 18$ \\
\hline $\begin{array}{r}43 \\
214\end{array}$ & $\begin{array}{l}98 \\
98\end{array}$ & $\begin{array}{l}H / 0 \\
H^{2} 2.25\end{array}$ & $\begin{array}{l}\text { HP } \\
\text { HP }\end{array}$ & $\begin{array}{l}1200 \\
1200\end{array}$ & $\begin{array}{l}0.501 \\
0.500\end{array}$ & $\begin{array}{l}200.0 \\
201.0\end{array}$ & $\begin{array}{l}170.8 \\
171.8\end{array}$ & $\begin{array}{l}632.0 \\
634.6\end{array}$ & $\begin{array}{l}0.398 \\
0.359\end{array}$ & .90 \\
\hline $\begin{array}{r}45 \\
161\end{array}$ & $\begin{array}{l}98 \\
98\end{array}$ & $\begin{array}{l}H / 0 \\
H \quad 2.25\end{array}$ & $\begin{array}{l}\text { HP } \\
\text { HP }\end{array}$ & $\begin{array}{l}12000 \\
1200\end{array}$ & $\begin{array}{l}0.246 \\
0.246\end{array}$ & $\begin{array}{l}400.7 \\
400.3\end{array}$ & $\begin{array}{l}377.2 \\
376.8\end{array}$ & $\begin{array}{l}803.5 \\
826.8\end{array}$ & $\begin{array}{l}0.163 \\
0.172\end{array}$ & 1.06 \\
\hline $\begin{array}{r}47 \\
163\end{array}$ & $\begin{array}{l}98 \\
98\end{array}$ & $\begin{array}{l}W / 0 \\
W^{2} 2.25\end{array}$ & $\begin{array}{l}H P \\
H P\end{array}$ & $\begin{array}{l}120.0 \\
120.0\end{array}$ & $\begin{array}{l}0.494 \\
0.497\end{array}$ & $\begin{array}{l}399.8 \\
400.4\end{array}$ & $\begin{array}{l}376.3 \\
376.9\end{array}$ & $\begin{array}{l}729.1 \\
716.9\end{array}$ & $\begin{array}{l}0.271 \\
0.262\end{array}$ & .97 \\
\hline $\begin{array}{r}49 \\
165\end{array}$ & $\begin{array}{l}98 \\
98\end{array}$ & $\begin{array}{l}W / C \\
H 2.25\end{array}$ & $\begin{array}{l}\text { HP } \\
H P\end{array}$ & $\begin{array}{l}1200 \\
1200\end{array}$ & $\begin{array}{l}0.923 \\
0.988\end{array}$ & $\begin{array}{l}401.1 \\
403.2\end{array}$ & $\begin{array}{l}377.7 \\
376.6\end{array}$ & $\begin{array}{l}676.2 \\
655.1\end{array}$ & $\begin{array}{l}0.428 \\
0.427\end{array}$ & 1.00 \\
\hline $\begin{array}{l}49 A \\
165\end{array}$ & $\begin{array}{l}98 \\
98\end{array}$ & $\begin{array}{l}W / 0 \\
H 2.25\end{array}$ & $\begin{array}{l}\text { MP } \\
\text { HP }\end{array}$ & $\begin{array}{l}1200 \\
1200\end{array}$ & $\begin{array}{l}0.989 \\
0.988\end{array}$ & $\begin{array}{l}401 \cdot 1 \\
400 \cdot 2\end{array}$ & $\begin{array}{l}377.6 \\
376.6\end{array}$ & $\begin{array}{l}678.6 \\
655.1\end{array}$ & $\begin{array}{l}0.462 \\
0.427\end{array}$ & . 92 \\
\hline $\begin{array}{r}52 \\
186\end{array}$ & $\begin{array}{l}98 \\
98\end{array}$ & $\begin{array}{l}H / 0 \\
H \quad 2.25\end{array}$ & $\begin{array}{l}\text { HP } \\
\text { HP }\end{array}$ & $\begin{array}{l}1200 \\
1200\end{array}$ & $\begin{array}{l}0.248 \\
0.251\end{array}$ & $\begin{array}{l}501.6 \\
493.6\end{array}$ & $\begin{array}{l}489.7 \\
487.4\end{array}$ & $\begin{array}{l}815.3 \\
842.8\end{array}$ & $\begin{array}{l}0.125 \\
0.139\end{array}$ & 1.11 \\
\hline $\begin{array}{r}54 \\
188\end{array}$ & $\begin{array}{l}98 \\
98\end{array}$ & $\begin{array}{l}H / 0 \\
w \quad 2.25\end{array}$ & $\begin{array}{l}\text { HP } \\
\text { HP }\end{array}$ & $\begin{array}{l}1200 \\
1200\end{array}$ & $\begin{array}{l}0.471 \\
0.495\end{array}$ & $\begin{array}{l}500.7 \\
500.4\end{array}$ & $\begin{array}{l}488.6 \\
488.3\end{array}$ & $\begin{array}{l}745.1 \\
759.6\end{array}$ & $\begin{array}{l}0.188 \\
0.209\end{array}$ & 1.11 \\
\hline $\begin{array}{r}56 \\
198\end{array}$ & $\begin{array}{l}98 \\
98\end{array}$ & $\begin{array}{l}H / 0 \\
H 2.25\end{array}$ & $\begin{array}{l}H P \\
H P\end{array}$ & $\begin{array}{l}1200 \\
1200\end{array}$ & $\begin{array}{l}1.008 \\
1.015\end{array}$ & $\begin{array}{l}500.3 \\
499.2\end{array}$ & $\begin{array}{l}488.2 \\
486.9\end{array}$ & $\begin{array}{l}716.2 \\
686.4\end{array}$ & $\begin{array}{l}0.357 \\
0.315\end{array}$ & .88 \\
\hline 58 & 98 & $\omega / 0$ & HP & 1200 & 2.021 & 500.7 & 488.6 & 665.9 & 0.556 & \\
\hline
\end{tabular}




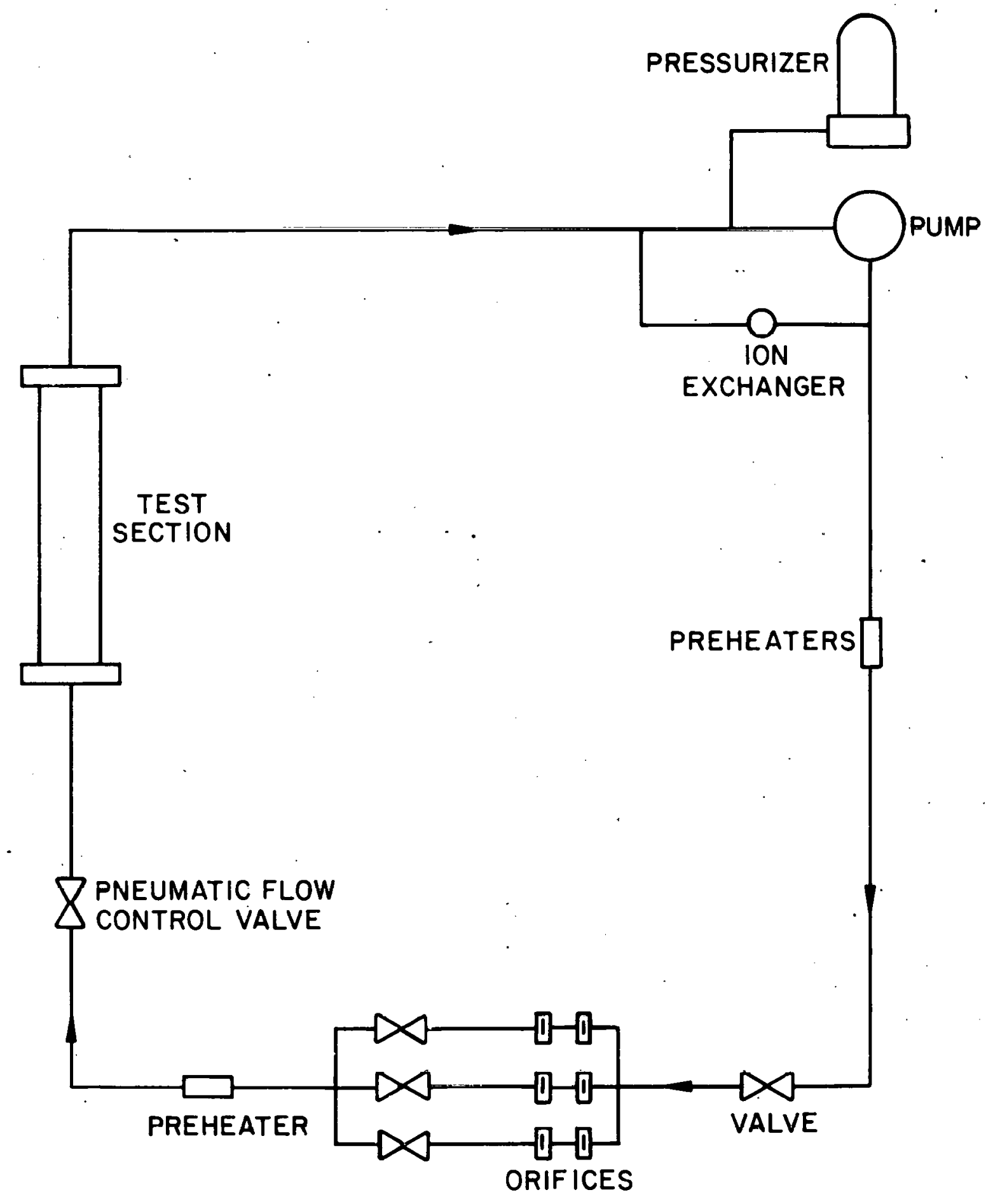

FIGURE I: SCHEMATIC DIAGRAM OF HOT PATCH TEST LOOP 


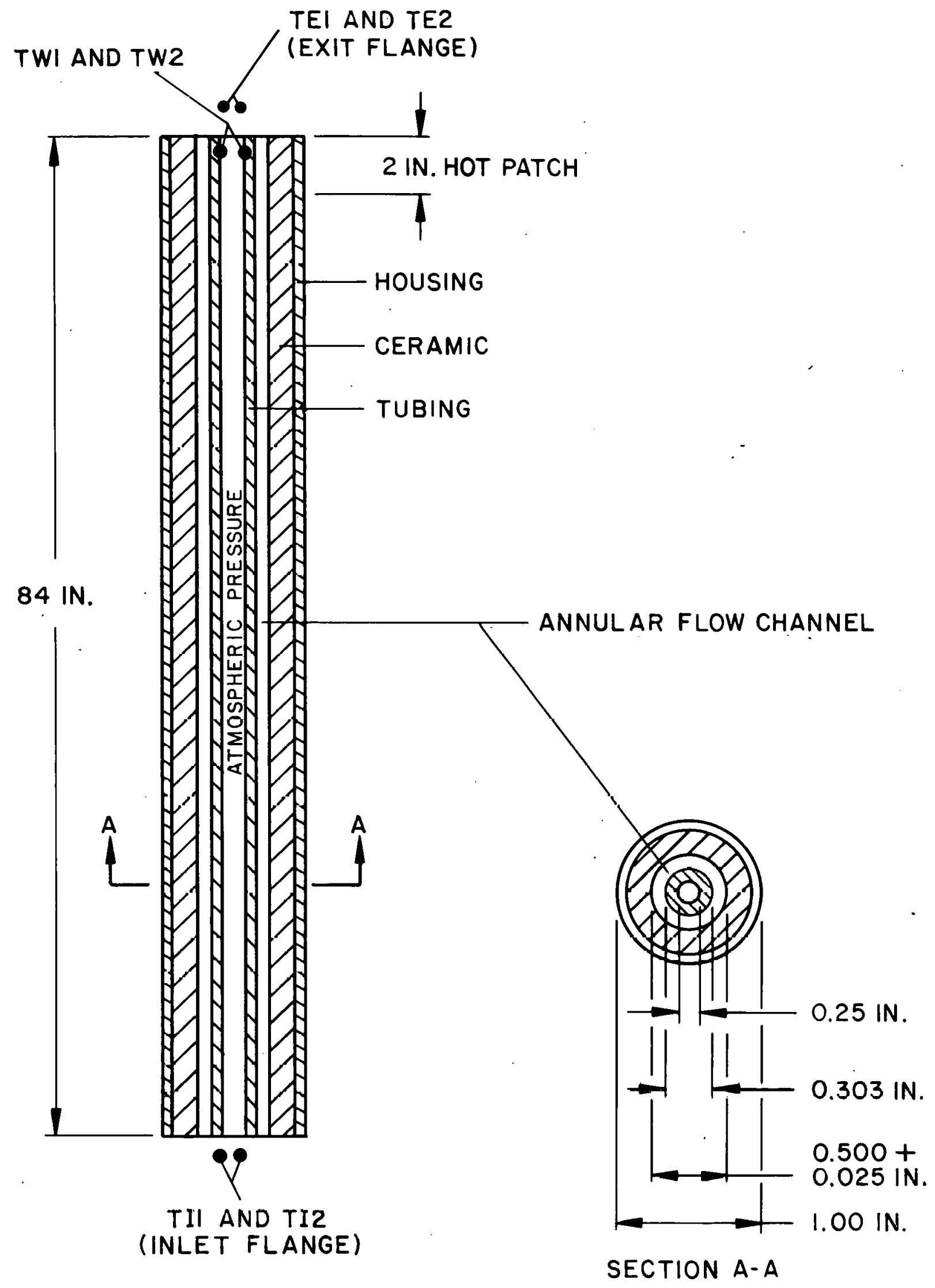

FIGURE 2: TEST SECTION AND INSTRUMENTATION 


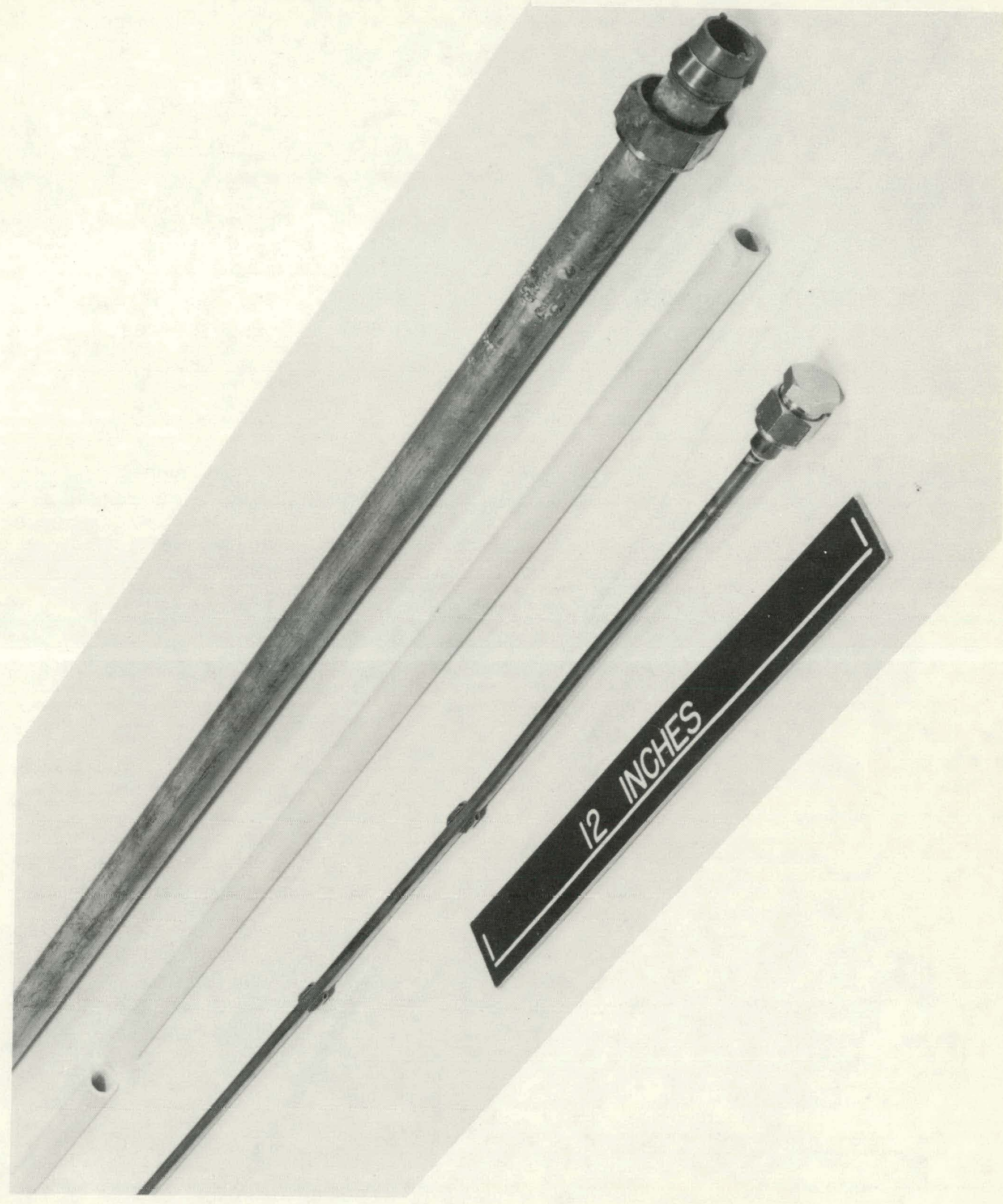

FIGURE 3: Partial Axial View of Hot Patch Test Section, Ceramic Housing and Backup Housing 


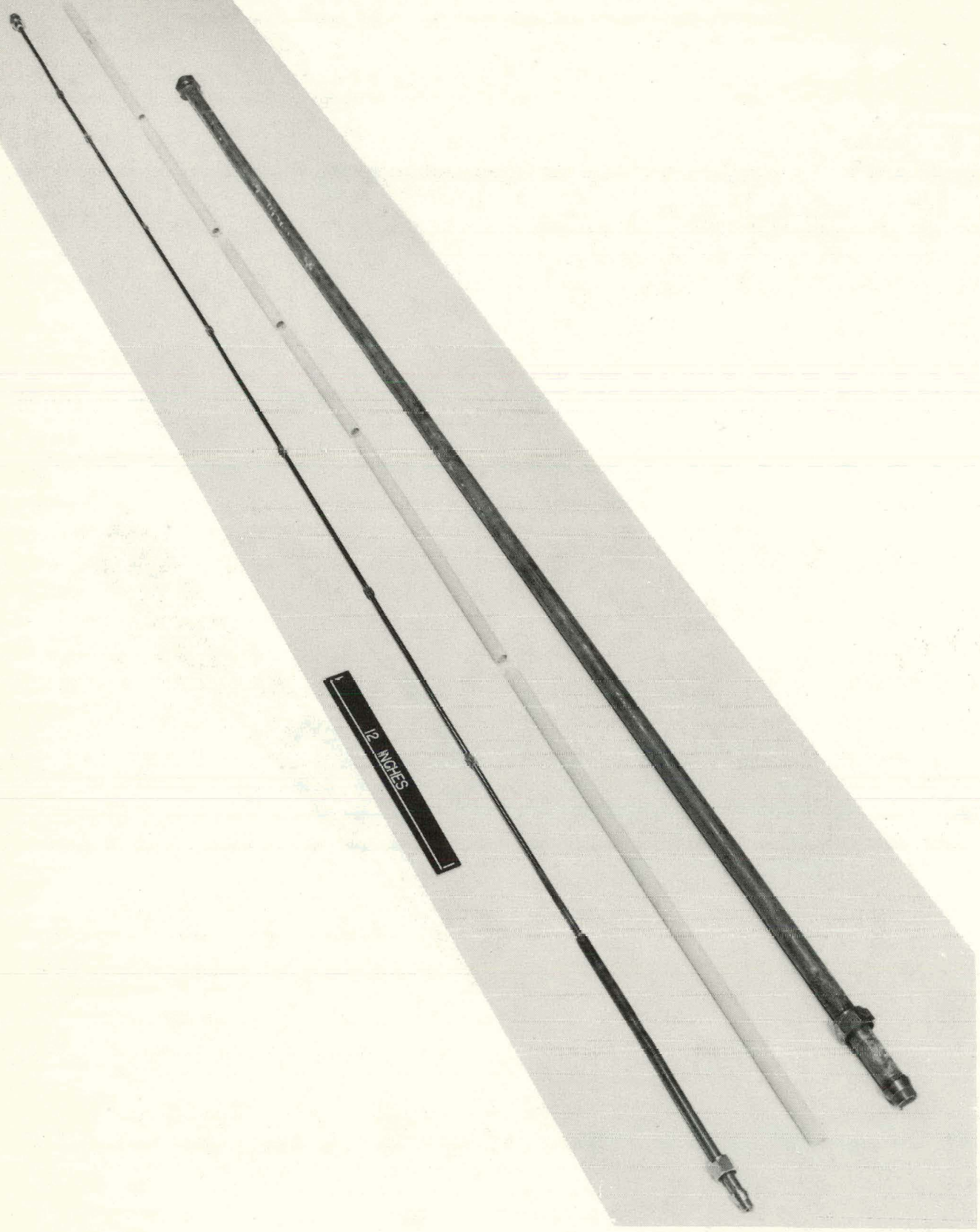

FIGURE 4. Full Axial View of Hot Patch Test Section, Ceramic Housing and Backup Housing 


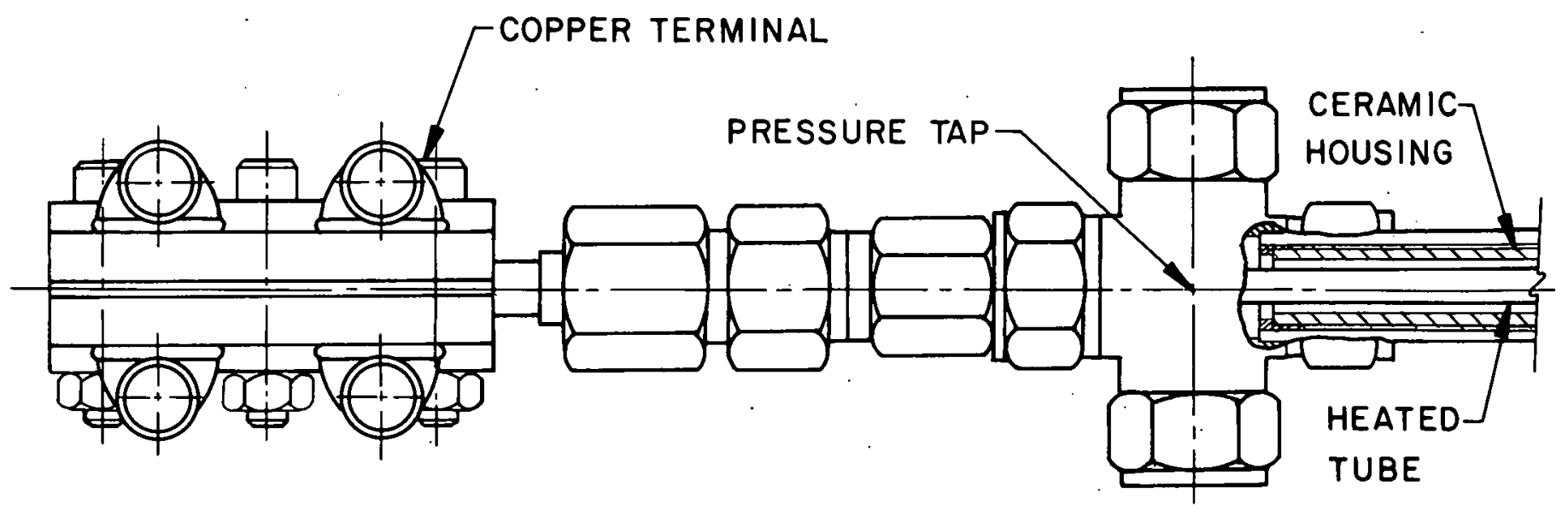

FIGURE 5. DETAILED SCHEMATIC OF INLET REGION OF HOT PATCH TEST SECTION

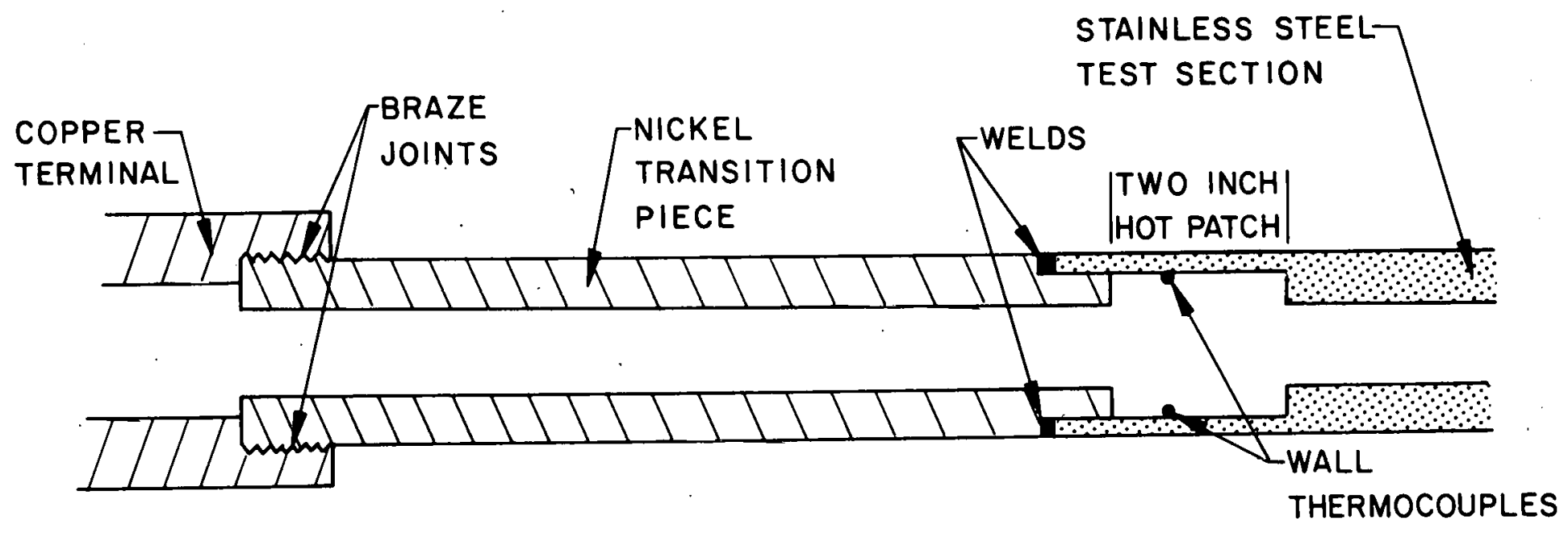

FIGURE 6. HEATED TUBE END CONNECTION DETAIL (NOT TO SCALE) 
$-32-$
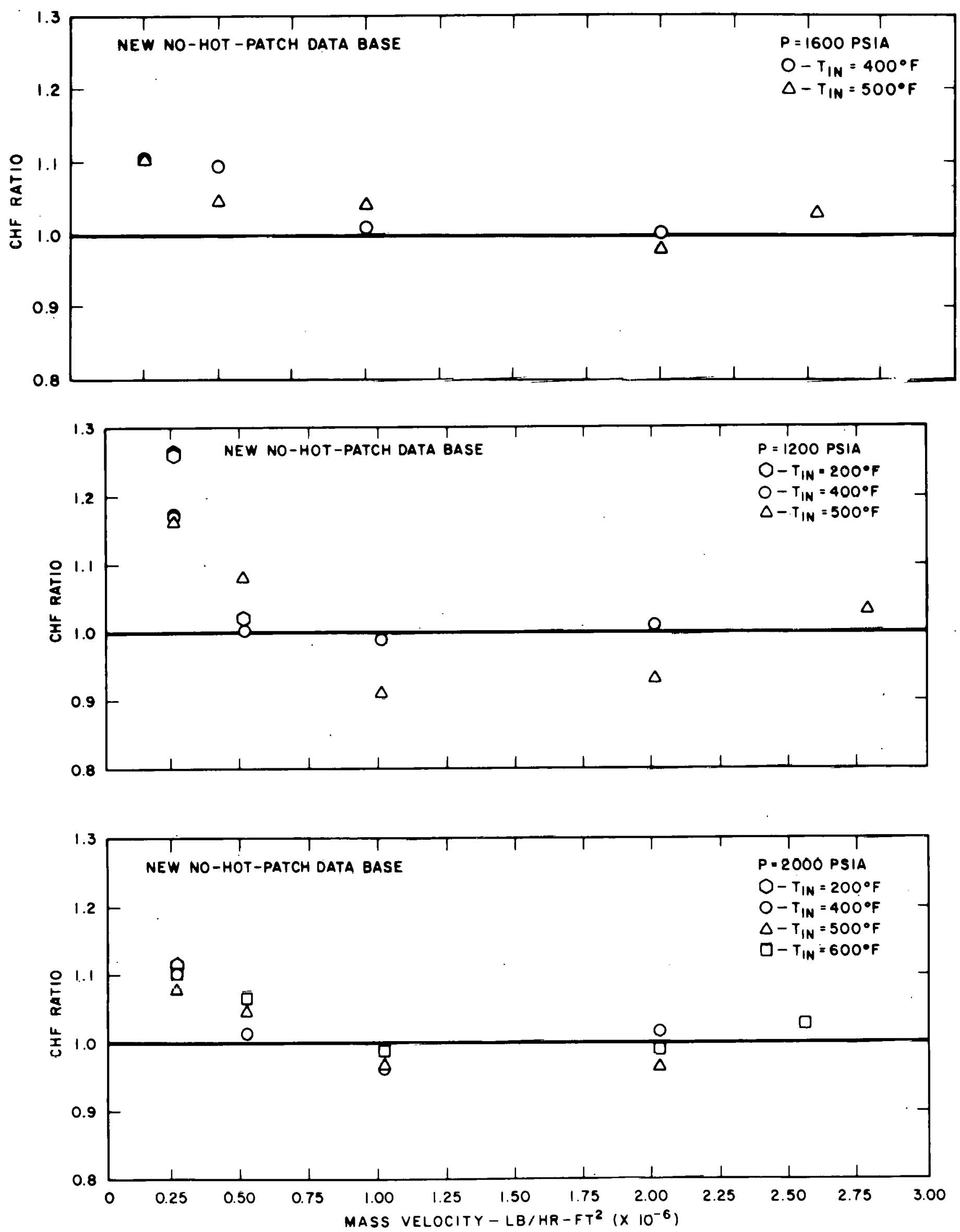

FIGURE 7: CHF RATIO DATA COMPARISON FOR HOT PATCH TEST

WITH I.5 HEAT FLUX RATIO 

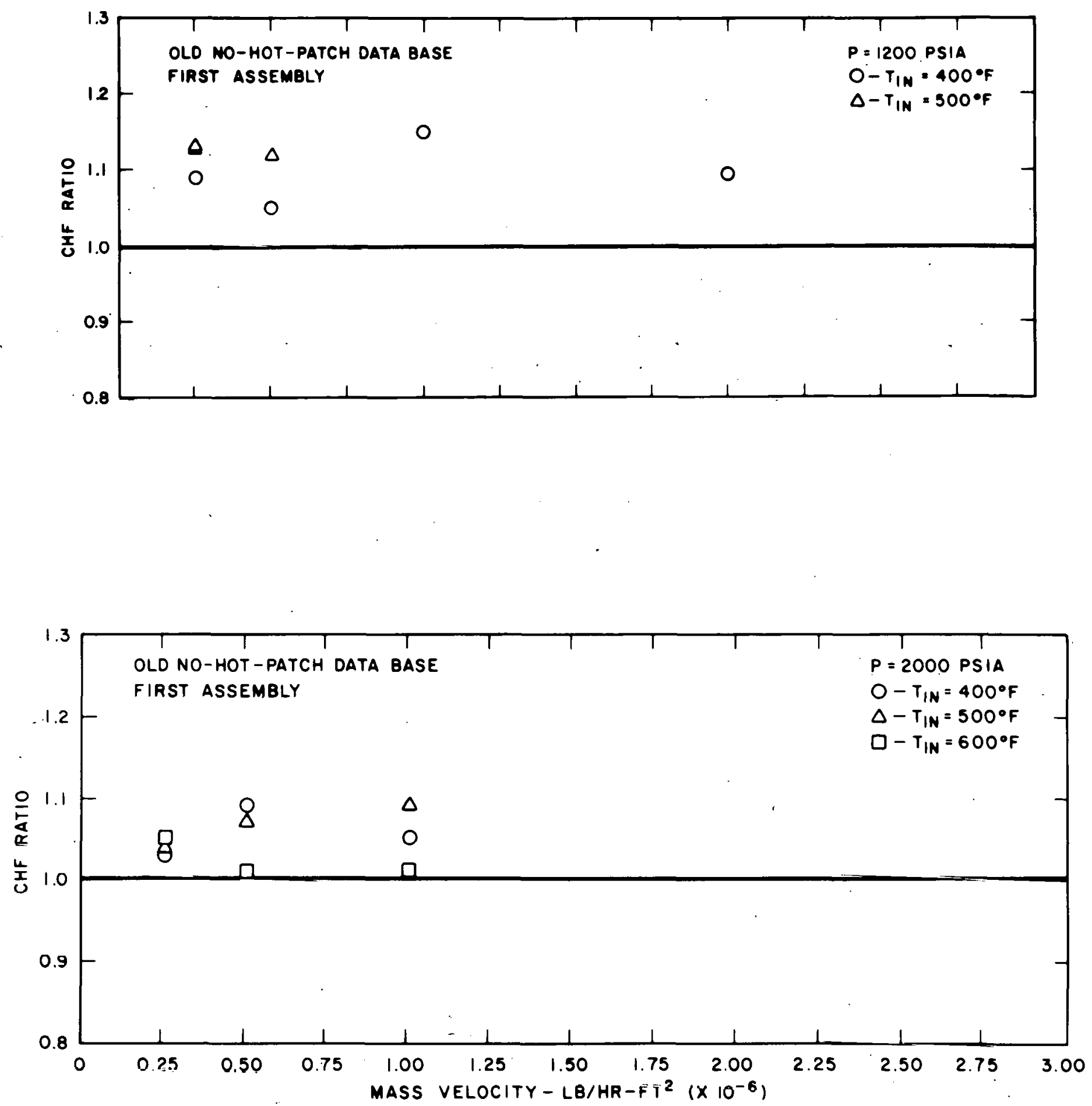

FIGURE 8: CHF RATIO DATA COMPARISON FOR 1.5 HOT PATCH TEST HEAT FLUX RATIO 

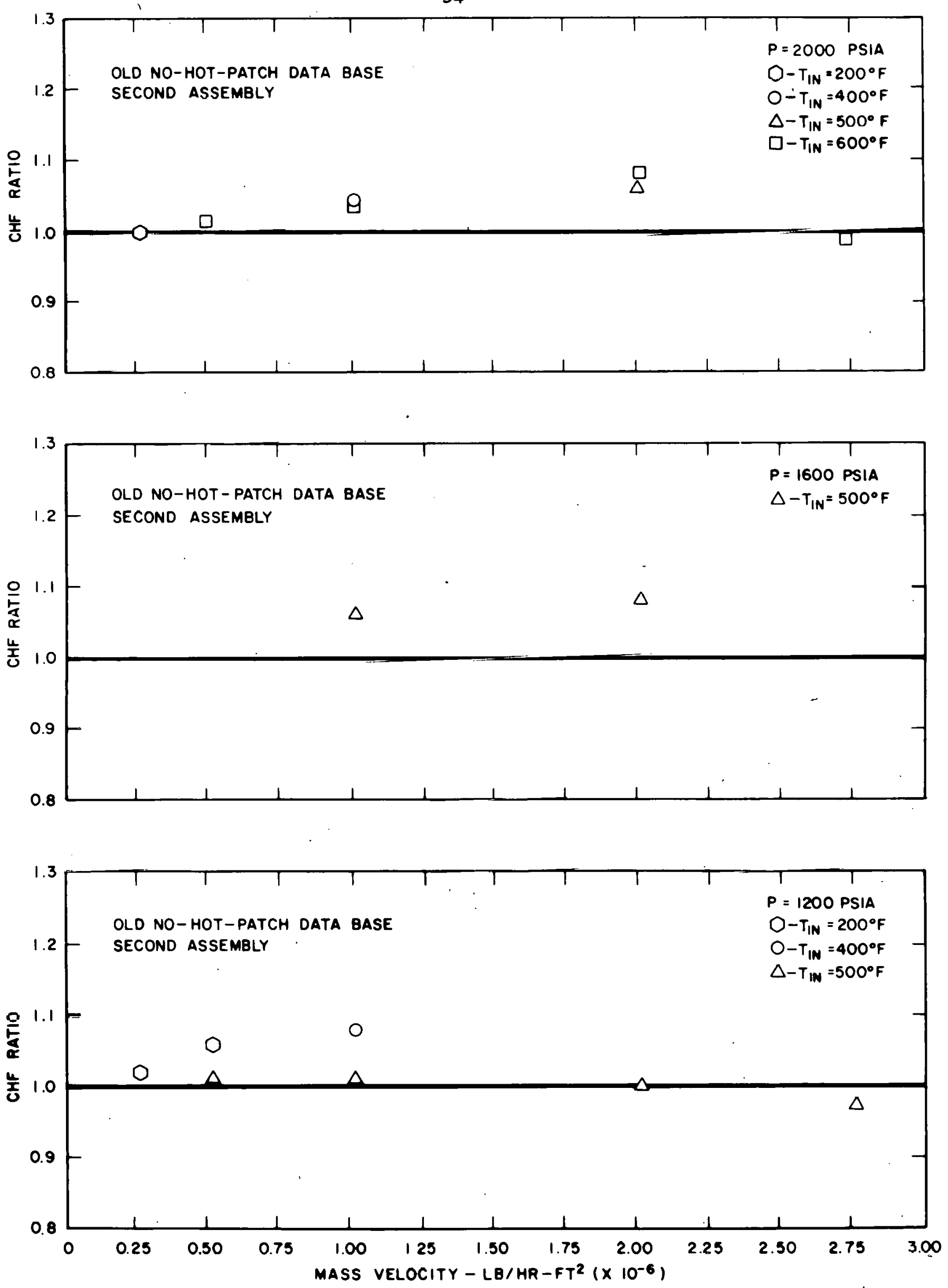

FIGURE 9. CHF RATIO DATA COMPARISON FOR I.5 HOT PATCH TEST 


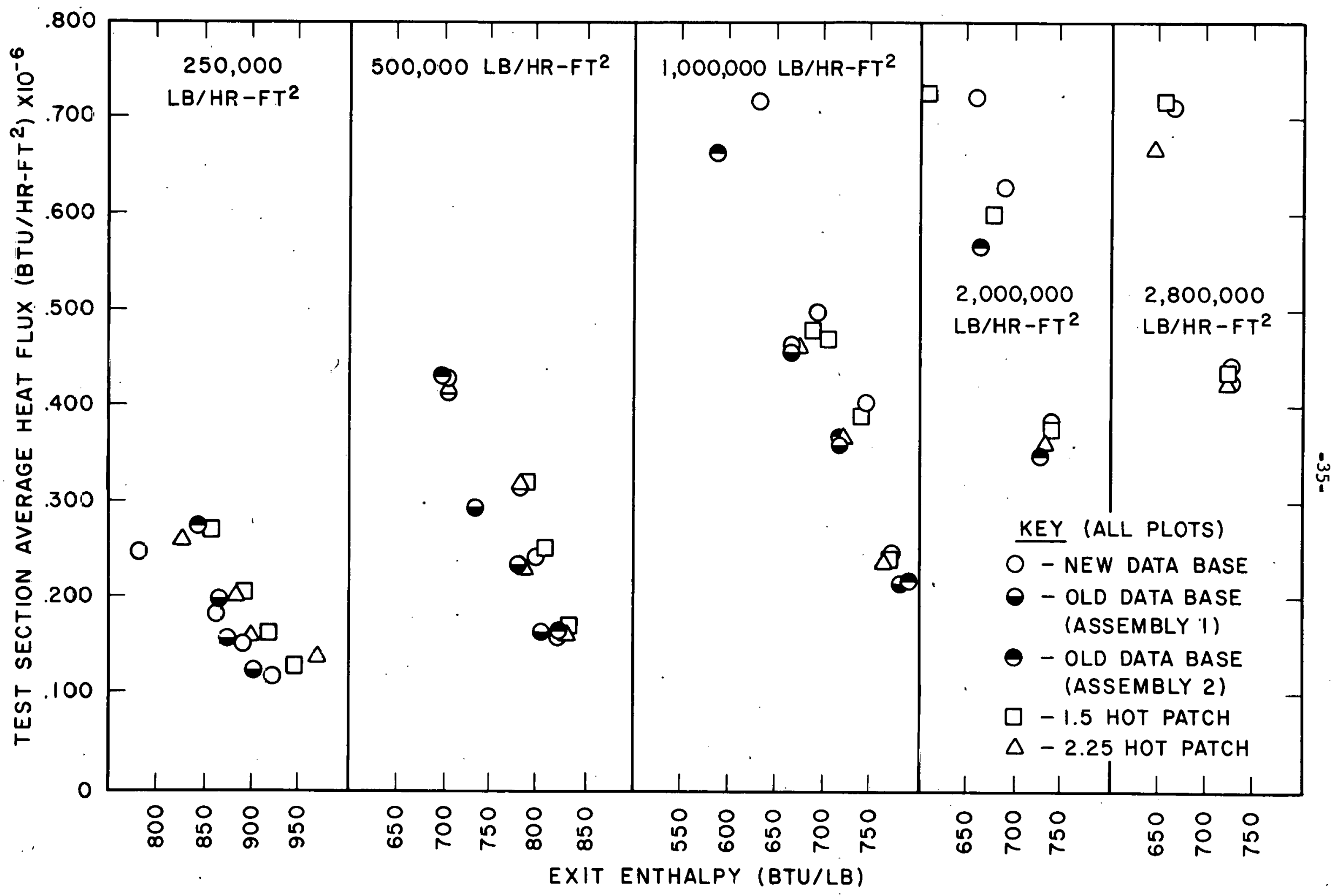

FIGURE 10: SUMMARY OF ALL CHF DATA AT 2000 PSIA 


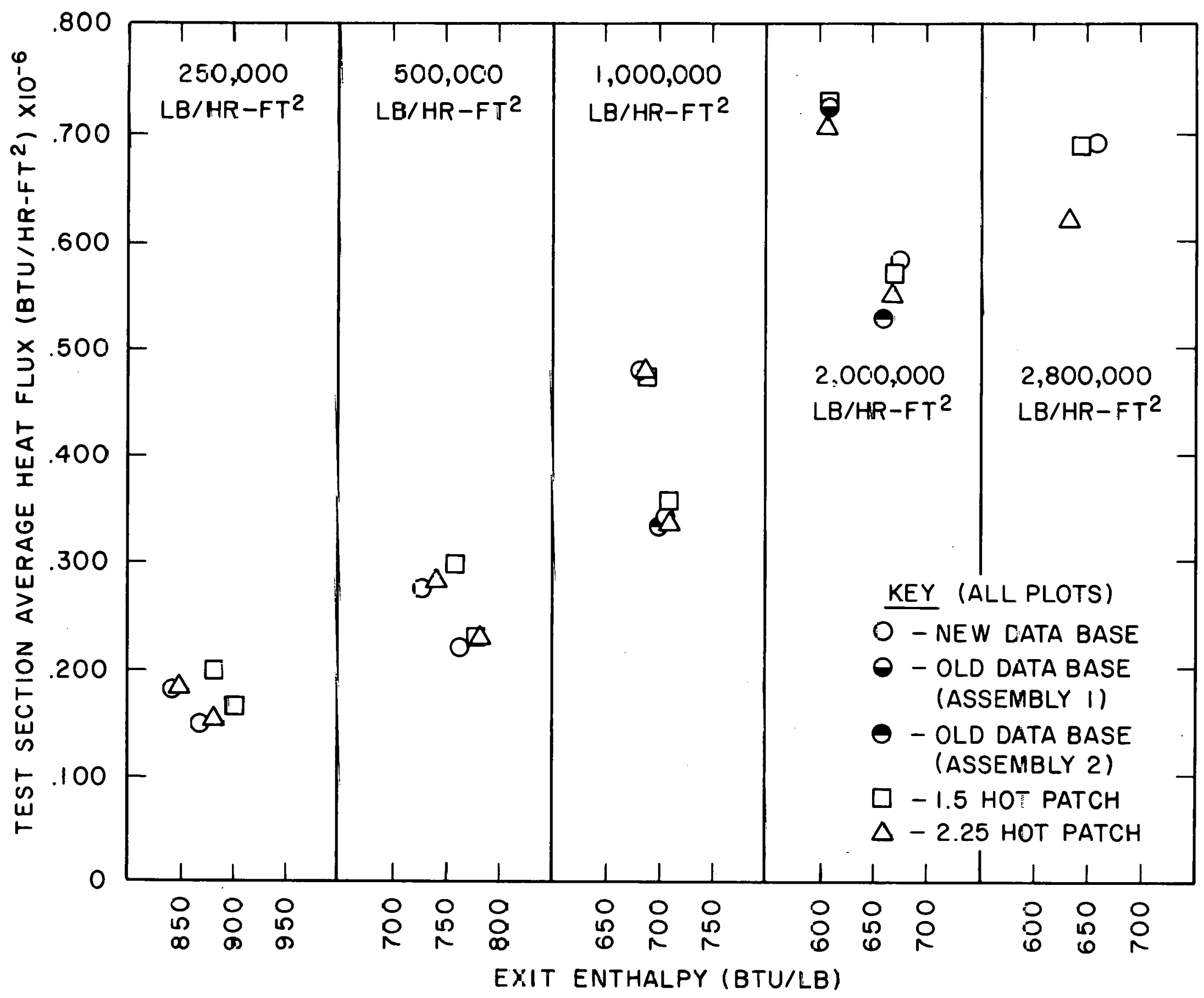

FIGURE 11: SUMMARY OF ALL CHF DATA AT 1600 PSIA 


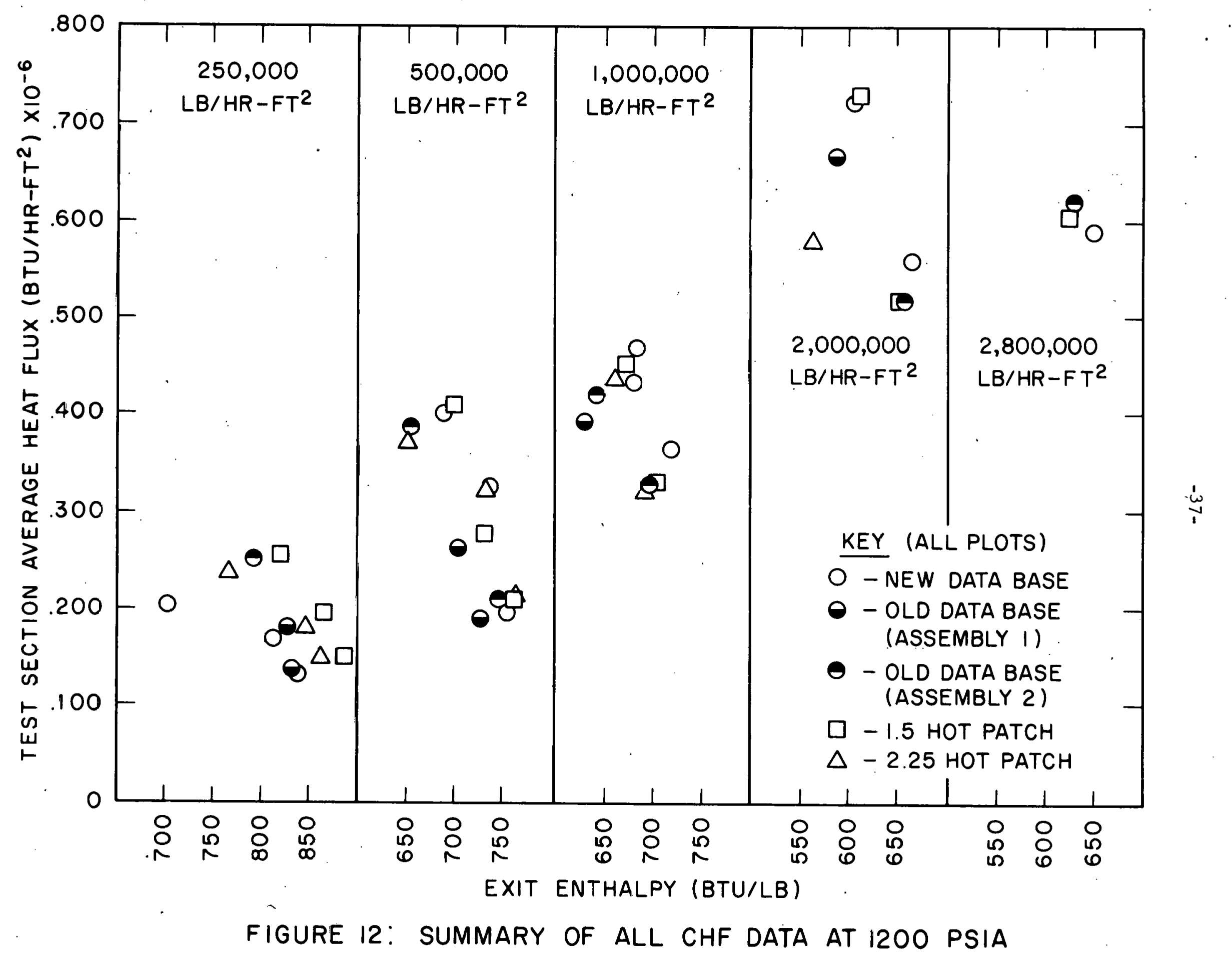



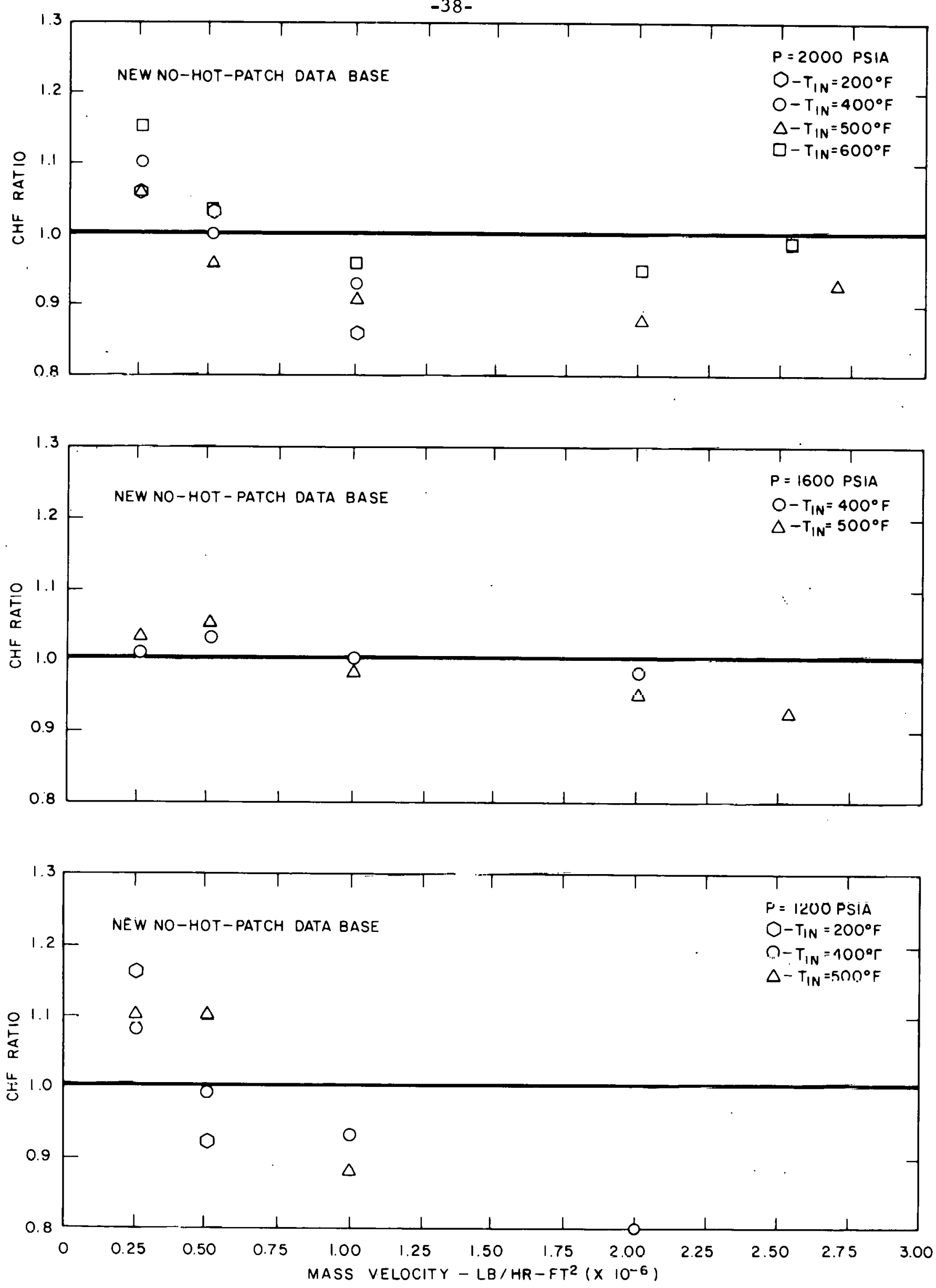

FIGURE 13. CHF RATIO DATA COMPARISON FOR 2.25 HOT PATCH TEST 

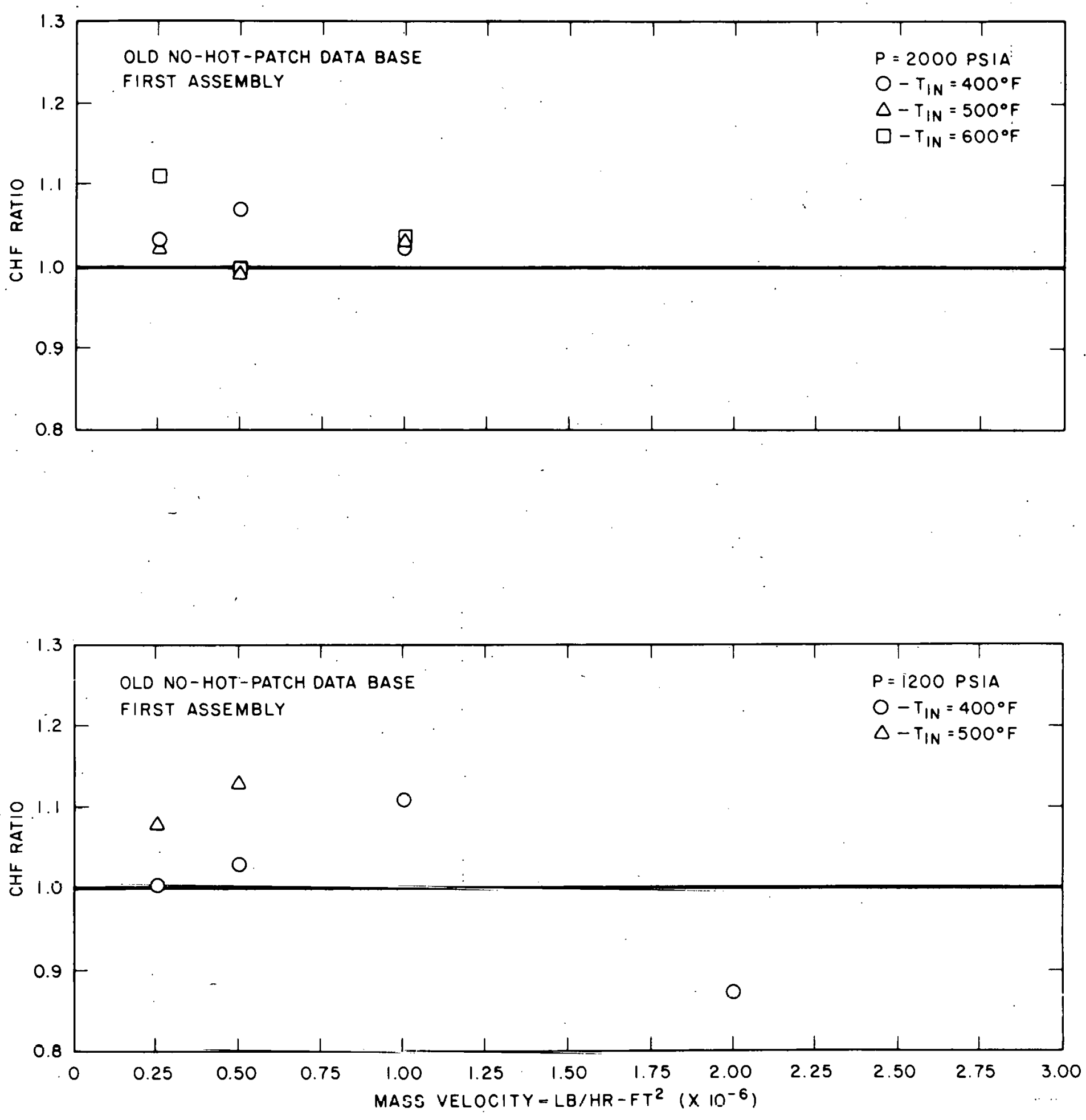

FIGURE 14: CHF RATIO DATA COMPARISON FOR 2.25 HOT PATCH TEST 
$-40-$
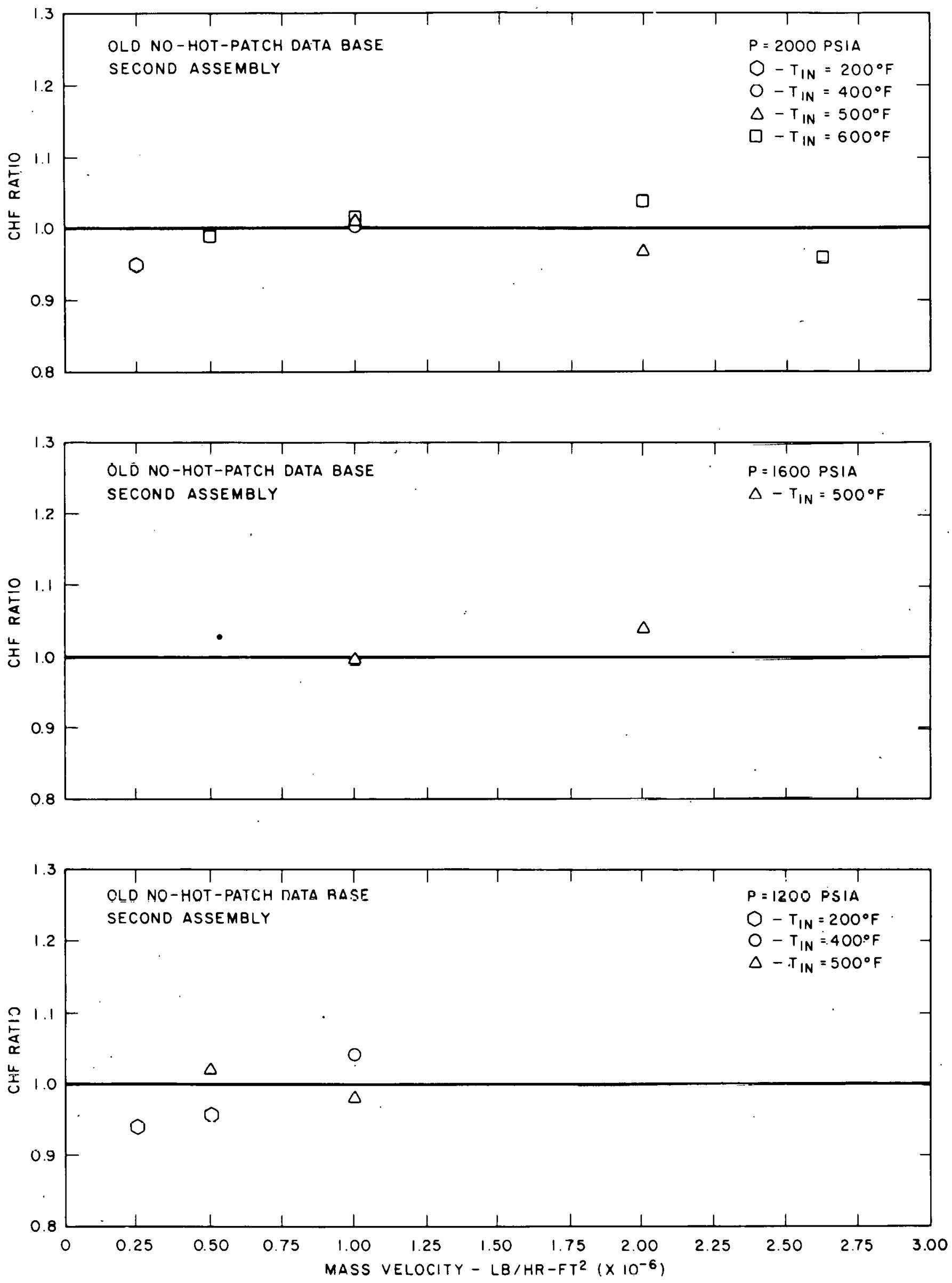

FIGURE 15: CHF RATIO DATA COMPARISON FOR 2.25 HOT PATCH TEST 


\section{Internally Heated Annulus CHF Experiments}

This Appendix describes critical heat flux experiments conducted in 1969 in the Thermal and Hydraulics Laboratory at Bettis Atomic Power Laboratory in which a single rod was used as an internally heated annulus. Tests were conducted in two assemblies which were nominally identical to each other and to the no-hot-patch test assembly described in this report.

The test sections were comprised of a 0.303 inch stainless steel rod with a 84 inch heated length, located inside of ceramic tubes (alumina) with a nominal 0.50 inch $I . D$. with vertical upflow of water. The rod was centered inside the ceramic tubes by double window spring cclilars similar to those described in Reference (a). The last spacer was located 9 inches upstream from the end of the heated length and additional spacers were located every 12 inches upstream.

Data were obtained over the following range of variables:

Pressure: $800,1200,1600$ and 2000 psia

Mass Velocity: $0.25 \times 10^{6}$ to $2.8 \times 10^{6} \mathrm{lb} / \mathrm{hr}-\mathrm{ft}^{2}$

Inlet Temperature: 200 to $600^{\circ} \mathrm{F}$

Heat Flux: $0.12 \times 10^{6}$ to $0.71 \times 10^{6} \mathrm{Btu} / \mathrm{hr}-\mathrm{ft}^{2}$

Table A presents the CHF data for assemblies 1 and 2. The test section insulation was judged to be sufficient to prevent appreciable heat loss and so no heat loss corrections were applied to the data.

\section{Reference}

(a) WAPD-TM-1013, "Critical Heat Fux and Pressure Drop Testing in Bundles of Twenty Rods," B. W. LeTourneau, et al, January 1975 
TABLE A

CHF Data With Last Spacer 9 Inches From the End of the Heated Length

\begin{tabular}{ccccc} 
& Mass & Inlet & & Exit \\
Pressure & Velocity & Enthalpy & Heat Flux & Enthalpy \\
P & $G \times 10^{-6}$ & $\mathrm{H}_{\text {in }}$ & $\emptyset \times 10^{-6}$ & $\mathrm{H}_{\text {ex }}$ \\
(psia) & $\left(1 \mathrm{~b} / \mathrm{hr}-\mathrm{ft} \mathrm{t}^{2}\right)$ & $(\mathrm{Btu} / \mathrm{b})$ & $\left(\mathrm{Btu} / \mathrm{hr}-\mathrm{ft}^{2}\right)$ & $(\mathrm{Btu} / \mathrm{b})$ \\
\hline
\end{tabular}

(FTRST ASSEMBLY)

$\begin{array}{llllll}345-04-1 & 2000 & 0.259 & 378 & 0.195 & 862 \\ 345-02-1 & 2000 & 0.260 & 485 & 0.165 & 871 \\ 345-03-7 & 2000 & 0.261 & 597 & 0.122 & 899 \\ 345-04-3 & 2000 & 0.520 & 377 & 0.292 & 738 \\ 345-02-3 & 2000 & 0.518 & 486 & 0.234 & 777 \\ 345-03-5 & 2000 & 0.521 & 601 & 0.165 & 805 \\ 345-08-3 & 2000 & 1.003 & 377 & 0.454 & 669 \\ 345-03-1 & 2000 & 1.003 & 487 & 0.356 & 716 \\ 345-03-3 & 2000 & 1.006 & 614 & 0.233 & 763 \\ 345-08-5 & 2000 & 1.501 & 377 & 0.627 & 645 \\ 34.5-04-7 & 1200 & 0.258 & 379 & 0.180 & 828 \\ 345-05-1 & 1200 & 0.258 & 488 & 0.137 & 830 \\ 345-04-5 & 1200 & 0.519 & 376 & 0.264 & 704 \\ 345-05-3 & 1200 & 0.516 & 488 & 0.190 & 726 \\ 345-07-5 & 1200 & 1.003 & 377 & 0.395 & 630 \\ 345-07-7 & 1200 & 1.501 & 379 & 0.536 & 608 \\ 345-08-1 & 1200 & 2.001 & 376 & 0.668 & 591 \\ 345-06-6 & 800 & 0.257 & 376 & 0.153 & 759 \\ 347-07-1 & 800 & 0.413 & 376 & 0.195 & 681 \\ 345-06-1 & 800 & 0.516 & 376 & 0.221 & 650 \\ 345-05-7 & 800 & 0.619 & 375 & 0.247 & 632 \\ 345-05-5 & 800 & 0.776 & 377 & 0.285 & 613 \\ 345-07-3 & 800 & 1.002 & 376 & 0.349 & 600\end{array}$

(SECOND ASSEMBLY)

$\begin{array}{llllll}345-20-5 & 2000 & 0.259 & 172 & 0.270 & 841 \\ 345-20-7 & 2000 & 0.519 & 173 & 0.425 & 701 \\ 345-18-1 & 2000 & 0.516 & 613 & 0.166 & 819 \\ 345-21-1 & 2000 & 1.004 & 171 & 0.658 & 592 \\ 345-19-4 & 2000 & 0.994 & 377 & 0.460 & 673 \\ 345-16-4 & 2000 & 1.005 & 490 & 0.362 & 721 \\ 345-17-7 & 2000 & 1.017 & 614 & 0.236 & 763 \\ 345-19-5 & 2000 & 1.506 & 377 & 0.616 & 641 \\ 345-16-5 & 2000 & 1.501 & 490 & 0.462 & 688 \\ 345-17-5 & 2000 & 1.522 & 613 & 0.304 & 741\end{array}$


TABLE A (Continued)

Mass Inlet

Velocity Enthalpy Heat Flux

Pressure

$G \times 10^{-6}$

$\mathrm{H}_{\text {in }}$

$\emptyset \times 10^{-6}$

Run No. $\quad$ (psia)

$\left(1 \mathrm{~b} / \mathrm{hr}-\mathrm{ft} \mathrm{t}^{2}\right)$

(Btu/1b) $\quad\left(B t u / h r-f t^{2}\right)$

Exit

Enthalpy

$\mathrm{H}$ ex

$(\mathrm{Btu} / 1 \mathrm{~b})$

\section{$345-16-7 \quad 2000$}

$345-17-3 \quad 2000$

2.012

2.024

0.564

0.350

664

484

2.709

613

1.002

484

0.444

727

$345-17-1 \quad 2000$

1.501

487

0.336

718

345-19-2 1600

1.998

490

0.446

700

345-19-1 1600

0.258

1.71

0.528

678

345-20-1 1200

0.516

172

0.251

660

345-20-3 1200

0.516

486

0.387

0.210

795

345-18-2 1200

1.004

1.004

374

487

0.422

0.329

374

345-19-6 1200

1.503

1.497

487

0.560

0.439

654

748

644

698

$345-18-4 \quad 1200$

1.999

488

0.520

614

675

345-18-5 1200

2.806

489

0.622

656

631 